\title{
Polycyclic aromatic hydrocarbon processing in interstellar shocks
}

\author{
E. R. Micelotta ${ }^{1,2}$, A. P. Jones ${ }^{2}$, and A. G. G. M. Tielens ${ }^{1,3}$ \\ 1 Sterrewacht Leiden, Leiden University, PO Box 9513, 2300 RA Leiden, The Netherlands \\ e-mail: micelot@strw. leidenuniv.nl \\ 2 Institut d'Astrophysique Spatiale, Université Paris Sud and CNRS (UMR 8617), 91405 Orsay, France \\ 3 NASA Ames Research Center, MS 245-3, Moffett Field, CA 94035, USA
}

Received 20 January 2009 / Accepted 16 October 2009

\section{ABSTRACT}

\begin{abstract}
Context. PAHs appear to be an ubiquitous interstellar dust component but the effects of shocks waves upon them have never been fully investigated.

Aims. We study the effects of energetic $(\approx 0.01-1 \mathrm{keV})$ ion $(\mathrm{H}, \mathrm{He}$ and $\mathrm{C})$ and electron collisions on PAHs in interstellar shock waves. Methods. We calculate the ion-PAH and electron-PAH nuclear and electronic interactions, above the threshold for carbon atom loss from a PAH, in $50-200 \mathrm{~km} \mathrm{~s}^{-1}$ shock waves in the warm intercloud medium.

Results. Interstellar PAHs $\left(N_{\mathrm{C}}=50\right)$ do not survive in shocks with velocities greater than $100 \mathrm{~km} \mathrm{~s}^{-1}$ and larger PAHs $\left(N_{\mathrm{C}}=200\right)$ are destroyed for shocks with velocities $\geq 125 \mathrm{~km} \mathrm{~s}^{-1}$. For shocks in the $\approx 75-100 \mathrm{~km} \mathrm{~s}^{-1}$ range, where destruction is not complete, the PAH structure is likely to be severely denatured by the loss of an important fraction (20-40\%) of the carbon atoms. We derive typical PAH lifetimes of the order of a few $\times 10^{8} \mathrm{yr}$ for the Galaxy. These results are robust and independent of the uncertainties in some key parameters that have yet to be well-determined experimentally.

Conclusions. The observation of PAH emission in shock regions implies that that emission either arises outside the shocked region or that those regions entrain denser clumps that, unless they are completely ablated and eroded in the shocked gas, allow dust and PAHs to survive in extreme environments.
\end{abstract}

Key words. shock waves - dust, extinction - supernovae: general

\section{Introduction}

Interstellar polycyclic aromatic hydrocarbon molecules (PAHs) are an ubiquitous component of the interstellar medium. The mid-infrared spectrum of the general diffuse interstellar medium as well as energetic environments near massive stars such as $\mathrm{H}$ II regions and reflection nebulae are dominated by broad emission features at 3.3, 6.2, 7.7, and $11.2 \mu \mathrm{m}$. These emission features are now generally attributed to infrared fluorescence by large PAH molecules containing 50-100 C-atoms, pumped by single FUV photons (see Tielens 2008, for a recent review). The observed spectra also show evidence for PAH clusters containing a few hundred C-atoms (Bregman et al. 1989; Rapacioli et al. 2005; Berné et al. 2007) as well as very small dust grains ( $230 \AA$ A Désert et al. 1990). It seems that the interstellar grain size distribution extends all the way into the molecular domain (Allamandola et al. 1989; Désert et al. 1990; Draine \& $\mathrm{Li}$ 2001). The origin and evolution of interstellar PAHs are somewhat controversial. On the one hand, based upon extensive laboratory studies of soot formation in terrestrial environments, detailed models have been made for the formation of PAHs in the ejecta of C-rich giants (Frenklach \& Feigelson 1989; Cherchneff et al. 1992) - as intermediaries or as sideproducts of the soot-formation process - and studies have suggested that such objects might produce enough PAHs to seed the ISM (Latter 1991). On the other hand, models have been developed where PAHs (as well as very small grains) are the byproduct of the grinding-down process of large carbonaceous grains in strong supernova shock waves which permeate the interstellar medium (Borkowski \& Dwek 1995; Jones et al. 1996). Grain-grain collisions shatter fast moving dust grains into small fragments and, for graphitic progenitor grains, these fragments might be more properly considered PAH molecules. The destruction of interstellar PAHs is equally clouded. Laboratory studies have shown that small (less than $16 \mathrm{C}$-atoms), (catacondensed) PAHs are rapidly photodissociated by $\sim 10 \mathrm{eV}$ photons (Jochims et al. 1994). However, this process is strongly size-dependent as larger PAHs have many more modes over which the internal energy can be divided and PAHs as large as $50 \mathrm{C}$-atoms might actually be stable against photodissociation in the ISM (Le Page et al. 2001; Allamandola et al. 1989). While strong shock waves have been considered as formation sites for interstellar PAHs, the destruction of these PAHs in the hot postshock gas has not been evaluated. Yet, high energy $(\sim 1 \mathrm{keV})$ collisions of PAHs with ions and electrons are highly destructive.

The observational evidence for PAHs in shocked regions is quite ambiguous. The majority of supernova remnants does not show PAH features (e.g. Cas A, Smith et al. 2009), but observations of N132D (Tappe et al. 2006) suggest the possibility of PAH survival in shocks. Recent work by Andersen et al. (2007) investigates the presence of PAHs in a subset of galactic supernova remnants detected in the GLIMPSE survey. Unfortunately the interpretation of such observations is not straightforward, because of the difficulty in disentangling the $\mathrm{PAH}$ features intrinsic to the shocked region with those arising from the surrounding material. Another interesting case is the starburst galaxy M 82, which shows above and below the galactic plane a huge bipolar outflow of shock-heated gas interwoven with PAH emis$\operatorname{sion}^{1}$ (Armus et al. 2007). PAHs have also been observed at high galactic latitudes in the edge-on galaxies NGC 5907 and

\footnotetext{
${ }^{1}$ http://chandra.harvard.edu/photo/2006/m82/
} 
NGC 5529 (Irwin \& Madden 2006; Irwin et al. 2007). Shock driven winds and supernovae can create a so-called "galactic fountain" (Bregman 1980) transporting material into the halo and these detections of PAHs suggests the possibility of survival or formation of the molecules under those conditions. On the other hand O'Halloran et al. $(2006,2008)$ have found a strong anti-correlation between the ratio $[\mathrm{FeII}] /[\mathrm{NeII}]$ and $\mathrm{PAH}$ strenght in a sample of low-metallicity starburst galaxies. Since [FeII] has been linked primarly to supernova shocks, the authors attributed the observed trend to an enhanced supernova activity which led to PAH destruction.

In our previous study (Jones et al. 1996), we considered the dynamics and processing of small carbon grains with $N_{\mathrm{C}} \geq 100$. The processing of these grains by sputtering (inertial and thermal) in ion-grain collisions and by vaporisation and shattering in grain-grain collisions was taken into account for all the considered grain sizes. In that work, the smallest fragments $\left(a_{\mathrm{f}}<5 \AA\right)$ were collected in the smallest size bin and not processed. In this work we now consider what happens to these smallest carbon grain fragments that we will here consider as PAHs. In this paper, we will consider relatively low velocity $\left(\leq 200 \mathrm{~km} \mathrm{~s}^{-1}\right)$ shocks where the gas cools rapidly behind the shock front but, because of their inertia, PAHs (and grains) will have high velocity collisions even at large postshock column densities. Collisions between PAHs and the gas ions occur then at the PAH velocity which will slowly decrease behind the shock front due to the gas drag. This relative velocity is thus independent of the ion mass and, for dust grains, destruction is commonly called inertial sputtering. Destruction of PAHs in high velocity $\left(\geq 200 \mathrm{~km} \mathrm{~s}^{-1}\right)$ shocks - which cool slowly through adiabatic expansion - is dominated by thermal sputtering and these shocks are considered in a subsequent paper (Micelotta et al. 2010, hereafter MJT).

This paper is organized as follows: Sect. 2 describes the theory of ion interaction with solids, Sect. 3 illustrates the application of this theory to PAH processing by shocks and Sect. 4 presents our results on PAH destruction. The PAH lifetime in shocks and the astrophysical implications are discussed in Sect. 5 and our conclusions summarized in Sect. 6.

\section{Ion interaction with solids}

\subsection{Nuclear interaction}

The approach used in our earlier work is not valid for planar PAH molecules with of the order of tens of carbon atoms. Here, we assume that collisions are binary in nature, as is assumed in work on solids (Lindhard et al. 1963, 1968; Sigmund 1981). If the energy transfer is above the appropriate threshold value, we assume that the carbon target is ejected from the molecule. For energy transfer below that threshold, the energy will become thermal energy and be radiated away.

In this description the "bulk" nature of the target enters only after the first interaction, when the projectile propagates into the material. We therefore consider only the first interaction, which is described in the binary collision approximation in a way that then conveniently allows us to take into account the "molecular" nature of the target.

In addition to the energy directly transferred to the target nucleus through elastic scattering (nuclear stopping or elastic energy loss), the energy loss to the atomic electrons (electronic stopping or inelastic energy loss) should also be considered (Lindhard et al. 1963, 1968). In a solid the energy transferred via electronic excitation is distributed around the impact region. For a PAH, which has a finite size, the energy will be spread out over the entire molecule. This energy will either be radiated away or a fragment can be ejected.

Nuclear and electronic stopping are simultaneous processes which can be treated separately (Lindhard et al. 1963). Figure 11 illustrates these effects and shows the PAH evolution following the loss of carbon atoms, $N_{\mathrm{C}}$ (lost), for the two limiting cases: 1) where there is an instantaneous and random removal of the lost carbon atoms and 2) where the carbon atoms are removed only from the periphery in order to preserve aromatic domain as much as possible. The reality of PAH erosion in shocks probably lies somewhere between these two extremes and will involve isomerisation and the formation of five-fold carbon rings that distort the structure from a perfectly two-dimensional form. This then begs the question as to the exact form and structure of small carbon species once growth resumes by atom insertion and addition. The full treatment of the nuclear stopping is given here, for the electronic stopping only the results of the calculations are shown, for the complete description of the phenomenon we refer the reader to paper MJT.

The treatment of PAH processing by shocks should also include the effects of fast electrons present in the gas. Because of their low mass, electrons can reach high velocities and hence high collision rates even at relatively low temperatures $\left(T \sim 10^{5} \mathrm{~K}\right)$, leading to potentially destructive collisions. Again for a detailed description of the electron-PAH interaction see paper MJT.

The theory of ion penetration into solids described here considers collisions where the transferred energy $T$ goes from 0 to the maximum transferable energy. For this study, we are interested in only those collisions that are able to remove carbon atoms from the PAH, i.e. for which the energy transferred is greater than the minimum energy $T_{0}$ required for $\mathrm{C}$ ejection. In Sect. 2.2 we present the modifications we introduce into the theory in order to treat the case of collisions above this threshold.

To describe the binary collision between a moving atom (or ion) and a stationary target atom (e.g. Sigmund 1981), a pure classical two-particle model using the Coulomb repulsion between the nuclei (Rutherford scattering) is adequate only at high energies, i.e. when $\varepsilon \gg 1$, where $\varepsilon$ is the dimensionless Lindhard's reduced energy

$\varepsilon=\frac{M_{2}}{M_{1}+M_{2}} \frac{a}{Z_{1} Z_{2} e^{2}} E$

where $M_{1}$ and $Z_{1}$ are the mass and atomic number of incident particle respectively, $M_{2}$ and $Z_{2}$ the mass and atomic number of target particle, $E$ is the kinetic energy of incident particle and $e$ is the electron charge, with $e^{2}=14.39 \mathrm{eV} \AA$. The quantity $a$ is the screening length, a parameter that defines the radial spread of the electronic charge about the nucleus. For the screening length we adopt the Universal Ziegler-Biersack-Littmark (ZBL) screening length $a_{\mathrm{U}}$ (Ziegler et al. 1985)

$a_{\mathrm{U}} \cong 0.885 a_{0}\left(Z_{1}^{0.23}+Z_{2}^{0.23}\right)^{-1}$

where $a_{0}=0.529 \AA$ is the Bohr radius. The condition $\varepsilon \gg 1$ implies that the energies are large enough that the nuclei approach closer to each other than the screening length $a$. At lower energies, $\varepsilon \lesssim 1$, it is essential to consider the screening of the Coulomb interaction. In this case the Rutherford approximation is not adequate and the scattering problem must be treated using a different approach.

To choose the appropriate formalism to describe our interaction, we need to calculate the reduced energy for our projectiles. For our study of the behaviour of PAHs in shocks, we 
Table 1. Kinetic energy $E$ and reduced energy $\varepsilon$ for $\mathrm{H}, \mathrm{He}$ and $\mathrm{C}$ impacting on a carbon atom.

\begin{tabular}{ccccc}
\hline \hline Projectile & $E^{a}\left(v_{\mathrm{p} 1}\right)$ & $\varepsilon^{b}\left(v_{\mathrm{p} 1}\right)$ & $E^{a}\left(v_{\mathrm{p} 2}\right)$ & $\varepsilon^{b}\left(v_{\mathrm{p} 2}\right)$ \\
\hline $\mathrm{H}$ & 7.30 & 0.031 & 117.4 & 0.50 \\
$\mathrm{He}$ & 29.4 & 0.048 & 469.7 & 0.76 \\
$\mathrm{C}$ & 88.1 & 0.028 & 1409. & 0.45 \\
\hline
\end{tabular}

Notes. (a) Kinetic energy in $\mathrm{eV}$. The projectile velocities $v_{\mathrm{p} 1}=$ $37.5 \mathrm{~km} \mathrm{~s}^{-1}$ and $v_{\mathrm{p} 2}=150 \mathrm{~km} \mathrm{~s}^{-1}$ are defined by the shock velocity $v_{\mathrm{S}}$ via the equation $v_{\mathrm{p}}=\frac{3}{4} v_{\mathrm{S}}$, with $v_{\mathrm{S}}=50$ and $200 \mathrm{~km} \mathrm{~s}^{-1}$ respectively.

(b) Dimensionless, calculated from Eqs. (1) and (2).

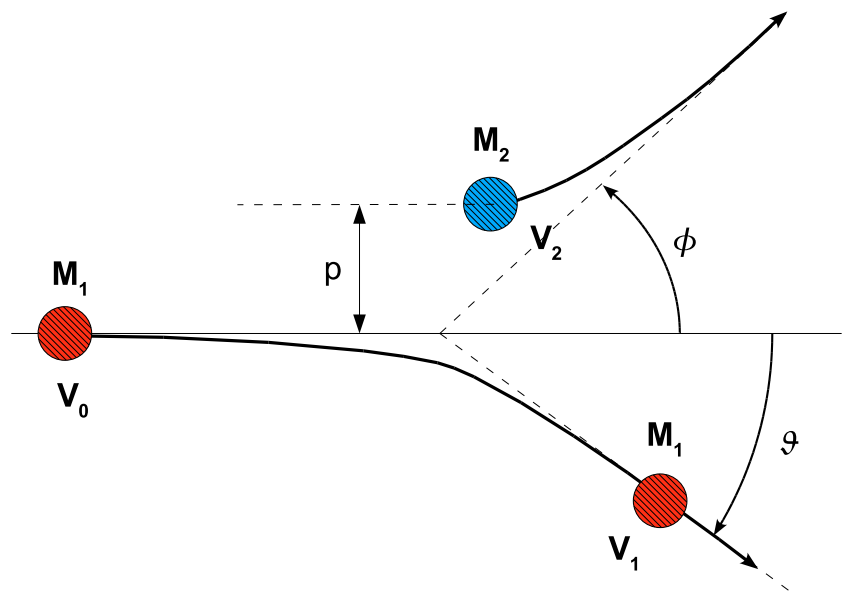

\section{LABORATORY COORDINATES}

Fig. 1. Scattering geometry for an elastic collision of particle 1 (mass $M_{1}$, initial velocity $v_{0}$, impact parameter $p$ ), on particle 2 (mass $M_{2}$, initial velocity zero). After the impact, the projectile particle 1 is deflected by the angle $\vartheta$ and continues its trajectory with velocity $v_{1}$. The target particle 2 recoils at an angle $\phi$ with velocity $v_{2}$.

consider the binary collision between $\mathrm{H}, \mathrm{He}$ and $\mathrm{C}$ ions (projectiles) and a carbon atom (target) in the PAH molecule. The velocity $v_{\mathrm{p}}$ of the projectile is determined by the shock velocity $v_{\mathrm{S}}$ through the relation $v_{\mathrm{p}}=\frac{3}{4} v_{\mathrm{S}}$. We consider here shock velocities between 50 and $200 \mathrm{~km} \mathrm{~s}^{-1}$. The corresponding projectile kinetic energies $E$ and reduced energies $\varepsilon$ are reported in Table 1 for the two limiting cases $v_{\mathrm{p} 1}=\frac{3}{4}(50)=37.5 \mathrm{~km} \mathrm{~s}^{-1}$ and $v_{\mathrm{p} 2}=\frac{3}{4}(200)=150 \mathrm{~km} \mathrm{~s}^{-1}$.

The calculation clearly shows that for the shocks we are considering $\varepsilon \lesssim 1$, implying that our problem cannot be treated in terms of Rutherford scattering but requires a different formalism, described by Sigmund (1981) and summarized below. The scattering geometry for an elastic collision of the projectile particle 1 on target particle 2 is illustrated in Fig. 1. Particle 1 has mass $M_{1}$, initial velocity $v_{0}$ and impact parameter $p$, where the impact parameter is the distance of closest approach of the centers of the two atoms/ions that would result if the projectile trajectory was undeflected. Particle 2 has mass $M_{2}$ and is initially at rest. After the impact, the projectile is deflected by the angle $\vartheta$ and continues its trajectory with velocity $v_{1}$. A certain amount of energy $T$ is transferred to the target particle which recoils at an angle $\phi$ with velocity $v_{2}$. The maximum transferable energy corresponds to a head-on collision (impact parameter $p=0$ ) and is given by

$T_{\mathrm{m}}=\gamma E=\frac{4 M_{1} M_{2}}{\left(M_{1}+M_{2}\right)^{2}} E$.
An important quantity to consider is the nuclear stopping cross section $S_{\mathrm{n}}(E)$, which is related to the average energy loss per unit path length of a particle travelling through a material of atomic number density $N$ (Lindhard et al. 1963)

$\frac{\mathrm{d} E}{\mathrm{~d} R}=N S_{\mathrm{n}}(E)=N \int \mathrm{d} \sigma(E, T) \cdot T$

where $\sigma(E, T)$ is the energy transfer cross section (see Appendix A for details). $S_{\mathrm{n}}(E)$ has the dimensions of (energy $\times$ area $\times$ atom $^{-1}$ ) and in fact represents the average energy transferred per atom in elastic collisions when summed over all impact parameters.

The nuclear stopping cross section can be expressed in terms of the Lindhard's reduced energy $\varepsilon$ and the dimensionless reduced nuclear stopping cross section $s_{\mathrm{n}}(\varepsilon)$ (Lindhard et al. 1968, see Eqs. (A.4) and (A.5)). For this latter we adopt the Universal reduced Ziegler-Biersack-Littmark (ZBL) nuclear stopping cross section $s_{\mathrm{n}}^{\mathrm{U}}$ (Ziegler et al. 1985), which is an analytical approximation to a numerical solution that reproduces well the experimental data. The ZBL reduced nuclear stopping cross section has the form

$s_{\mathrm{n}}^{\mathrm{U}}(\varepsilon)= \begin{cases}\frac{0.5 \ln (1+1.1383 \varepsilon)}{\varepsilon+0.01321 \varepsilon^{0.21226}+0.19593 \varepsilon^{0.5}} & \varepsilon \leq 30 \\ \frac{\ln \varepsilon}{2 \varepsilon} & \varepsilon>30\end{cases}$

and the nuclear stopping cross section $S_{\mathrm{n}}(E)$ can be written as

$S_{\mathrm{n}}(E)=4 \pi a_{\mathrm{U}} Z_{1} Z_{2} e^{2} \frac{M_{1}}{M_{1}+M_{2}} s_{\mathrm{n}}^{\mathrm{U}}(\varepsilon)$

with the screening length $a_{\mathrm{U}}$ from Eq. (2).

\subsection{Nuclear interaction above threshold}

For this study we are interested in destructive collisions, i.e., collisions for which the average transferred energy $T$ exceeds the minimum energy $T_{0}$ required to remove a carbon atom from the PAH. The theory discussed in Sect. 2.1 does not treat this situation and considers the specific case where $T_{0}=0$ (no threshold). To include the treatment of collisions above threshold $\left(T_{0}>0\right)$ we developped the appropriate expressions for the relevant quantities described in the previous sections.

The definition of the nuclear stopping cross section $S_{\mathrm{n}}(E)$ can be written in a more general way as

$S_{\mathrm{n}}(E)=\int_{T_{0}}^{T_{\mathrm{m}}} \mathrm{d} \sigma(E, T) \cdot T$

where $T_{0} \geq 0$. The total energy transfer cross section per carbon atom $\sigma(E)$ is defined by

$\sigma(E)=\int_{T_{0}}^{T_{\mathrm{m}}} \mathrm{d} \sigma(E, T)$.

In this case the threshold $T_{0}$ must be strictly positive, otherwise $\sigma$ would diverge (this can be verified by substituting the expression for $\mathrm{d} \sigma$ from Eq. (A.1) and evaluating the integral). Finally, the average energy transferred in a binary collision is given by the ratio between $S_{\mathrm{n}}$ and $\sigma$

$\langle T(E)\rangle=\frac{S_{\mathrm{n}}(E)}{\sigma(E)}$. 
Table 2. Threshold energy $T_{0}$ and critical kinetic energy $E_{0 \mathrm{n}}$ for $\mathrm{H}, \mathrm{He}$ and $\mathrm{C}$ ions impacting on a carbon atom.

\begin{tabular}{cccc}
\hline \hline$T_{0}$ & $E_{0 \mathrm{n}}(\mathrm{H})$ & $E_{0 \mathrm{n}}(\mathrm{He})$ & $E_{0 \mathrm{n}}(\mathrm{C})$ \\
\hline 4.5 & 15.8 & 6.0 & 4.5 \\
7.5 & 26.4 & 10. & 7.5 \\
10. & 35.2 & 13. & 10. \\
12. & 42.3 & 16. & 12. \\
15. & 52.8 & 20. & 15. \\
\hline
\end{tabular}

Notes. $T_{0}$ and $E_{0 \mathrm{n}}$ in $\mathrm{eV}$.

The condition $\gamma E=T_{\mathrm{m}}>T_{0}=\gamma E_{0 \mathrm{n}}$ then imposes, for the kinetic energy of the incoming ion, the condition that $E>E_{0 \text { n }}=$ $T_{0} / \gamma$. In Table 2 we report the critical energies $E_{0 \mathrm{n}}$ for $\mathrm{H}, \mathrm{He}$ and $\mathrm{C}$ ions corresponding to different values of the threshold energy $T_{0}$.

Using $\mathrm{d} \sigma$ from Eq. (A.1) and evaluating the above integrals we obtain

$$
\begin{aligned}
& S_{\mathrm{n}}(E)=\frac{C_{m} E^{-m}}{1-m}\left[T_{\mathrm{m}}^{1-m}-T_{0}^{1-m}\right] \\
& \sigma(E)=\frac{C_{m} E^{-m}}{m}\left[T_{0}^{-m}-T_{\mathrm{m}}^{-m}\right] \\
& \langle T(E)\rangle=\frac{m}{1-m} \frac{T_{\mathrm{m}}^{1-m}-T_{0}^{1-m}}{T_{0}^{-m}-T_{\mathrm{m}}^{-m}} .
\end{aligned}
$$

To calculate the quantity $m=m(E)$ we use the following expression from Ziegler et al. (1985)

$m(E)=1-\exp \left[-\exp \sum_{i=0}^{5} a_{i}\left(0.1 \ln \left(\frac{\varepsilon(E)}{\varepsilon_{1}}\right)^{i}\right)\right]$

with $\varepsilon_{1}=10^{-9}$ and $a_{i}=-2.432,-0.1509,2.648,-2.742$, $1.215,-0.1665$.

Combining Eqs. (A.3) and (A.4), after some algebraic manipulation, we can rewrite the above expressions for $S_{\mathrm{n}}, \sigma$ and $\langle T\rangle$ in the more convenient form shown below. The full calculation is reported in Appendix A. As explained in Sect. 2.1, we adopt for the reduced stopping cross section the ZBL function $s_{\mathrm{n}}^{\mathrm{U}}(\varepsilon)\left(\right.$ Eq. (5)) with the appropriate screening length $a_{\mathrm{U}}$.

$$
\begin{aligned}
& S_{\mathrm{n}}(E)=4 \pi a Z_{1} Z_{2} e^{2} \frac{M_{1}}{M_{1}+M_{2}} s_{\mathrm{n}}^{\mathrm{U}}(\varepsilon)\left[1-\left(\frac{E_{0 \mathrm{n}}}{E}\right)^{1-m}\right] \\
& \sigma(E)=4 \pi a Z_{1} Z_{2} e^{2} \frac{M_{1}}{M_{1}+M_{2}} s_{\mathrm{n}}^{\mathrm{U}}(\varepsilon) \frac{1-m}{m} \frac{1}{\gamma E}\left[\left(\frac{E_{0 \mathrm{n}}}{E}\right)^{-m}-1\right] \\
& \langle T(E)\rangle=\frac{m}{1-m} \gamma \frac{E^{1-m}-E_{0 \mathrm{n}}^{1-m}}{E_{0 \mathrm{n}}^{-m}-E_{m}^{-m}} .
\end{aligned}
$$

Note that the term outside of the square brackets in Eqs. (14) and (15) is the stopping cross section $S_{\mathrm{n}}(E)$ when $T_{0}=0$ (no threshold).

The nuclear stopping cross section $S_{\mathrm{n}}(E)\left(\mathrm{eV} \AA^{2}\right.$ atom $\left.^{-1}\right)$, the total energy transfer cross section $\sigma(E)\left(\AA^{2}\right.$ atom $\left.^{-1}\right)$ and the average energy transferred $\langle T(E)\rangle(\mathrm{eV})$, for $\mathrm{H}$, He and $\mathrm{C}$ ions impacting on a carbon atom, calculated from the above expressions assuming a threshold $T_{0}=7.5 \mathrm{eV}$, are shown in Fig. 2 .

The sharp cut on the left-hand side of the curves arises from the fact that we are treating collisions above threshold, and these quantities are defined only for energies of the incident ion greater than the critical value $E_{0 n}$. It can be seen that all quantities increase in absolute value with increasing atomic number and mass of the projectile $\left(Z_{1}\right.$ and $\left.M_{1}\right)$. The two vertical lines indicate the minimum and maximum kinetic energy of the projectile considered in our study, corresponding to the PAH velocity in the 50 and $200 \mathrm{~km} \mathrm{~s}^{-1}$ shocks respectively. The values are those calculated in Sect. 2.1 and reported in Table 1. The figure cleary shows that for hydrogen the critical value $E_{0 n}$ is greater than the lower limit of energy range. This implies that in the lower velocity shocks hydrogen is not energetic enough to cause carbon ejection. The curves for $S_{\mathrm{n}}$ presents a characteristic convex shape with a maximum, illustrating that nuclear energy transfer is important only for projectiles with energy falling in a specific range. In particular, the nuclear stopping becomes zero at high energies, with a limiting value depending on projectile and target: in our case, going from $\mathrm{H}$ to $\mathrm{C}$ impacting on carbon, the curves extend further to the right, in the direction of higher energies. In the high energy regime, the energy transfer is dominated by electronic stopping (MJT).

For a given incident ion energy, the difference between the values of $S_{\mathrm{n}}$ in the threshold and no-threshold cases results from the definition of the nuclear stopping and from the properties of $\mathrm{d} \sigma$. The differential cross section (cf. Eq. (A.1)) strongly prefers collisions with low energy transfers $\left(T \ll T_{\mathrm{m}}\right)$ and, moreover, decreases in absolute magnitute with increasing $E$. For each $E, S_{\mathrm{n}}$ is defined as the integral over the transferred energy $T$, of the product between $T$ and the corresponding cross section $\mathrm{d} \sigma$. Choosing $T_{0}>0$ means excluding from the integral all energy transfers $T<T_{0}$, for which the cross section has the highest values. The remaining terms have higher values of $T$ but lower values of $\mathrm{d} \sigma$, then the integral gives a result smaller than the no-threshold case, which includes all small energy transfers with their higher cross sections.

The total cross section $\sigma(E)$ clearly shows that the projectiles can efficiently transfer energy to the target atom only when their kinetic energy lies in the appropriate window. In particular, it can be seen that the average energy transferred $\langle T\rangle$ increases with $E$, nevertheless at high energies $\sigma$ is close to zero (and the collision rate will be small). For a fixed target atom (in our case, carbon), the width of the $\sigma$ curve, and consequently the width of the energy window, increases with $Z$ and $M$ of the projectile. Heavier ions transfer more energy and in a more efficient way.

\subsubsection{The threshold energy $T_{0}$}

The threshold energy, $T_{0}$, is the minimum energy that must be transferred via nuclear excitation to a carbon atom, in order to eject that same atom from the PAH molecule. The choice for $T_{0}$ for a PAH is unfortunately not well-constrained. There are no experimental determinations, and the theoretical evaluation is uncertain. The analog of $T_{0}$ in a solid is the displacement energy $T_{\mathrm{d}}$, defined as the minimum energy that one atom in the lattice must receive in order to be moved more than one atomic spacing away from its initial position, to avoid the immediate hop back into the original site. For graphite, the data on the threshold energy for atomic displacement differ significantly, varying from $\sim 30 \mathrm{eV}$ (Montet 1967; Montet \& Myers 1971) to $12 \mathrm{eV}$ (Nakai et al. 1991) largely depending on direction (e.g., within or perpendicular to the basal plane). For a PAH, the lower value (corresponding to the perpendicular direction) seems then more appropriate. For amorphous carbon, Cosslett (1978) has found a low value of $5 \mathrm{eV}$. Electron microscopy studies by Banhart (1997) on graphitic nanostructures irradiated with electrons of different energies, indicate that a 
E. R. Micelotta et al.: PAH processing in interstellar shocks
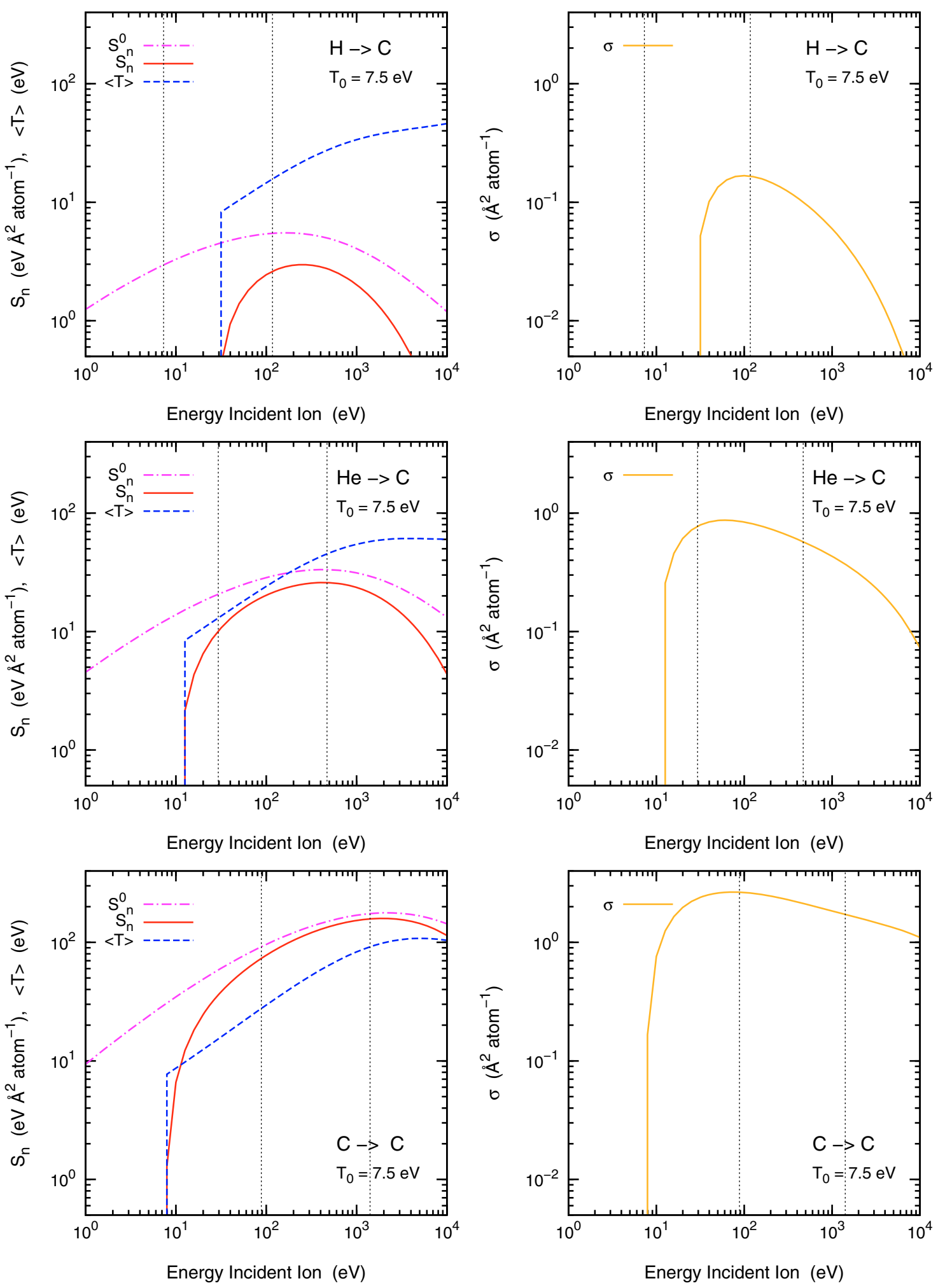

Fig. 2. The nuclear stopping cross section $S_{\mathrm{n}}(E)$, the total cross section $\sigma(E)$ and the average energy transferred $\langle T(E)\rangle$ calculated for $\mathrm{H}$, He and C ions impacting on a carbon atom. The curves are calculated for the threshold energy $T_{0}=7.5 \mathrm{eV}$. The nuclear stopping cross section $S_{\mathrm{n}}^{0}$ corresponding to $T_{0}=0$ (no threshold) is shown for comparison. The two vertical lines indicate the limiting energies for the incident ion. These are defined as the kinetic energies of the projectile when its velocity $v_{\mathrm{p}}$ equals $\frac{3}{4}\left(v_{\mathrm{S} 1, \mathrm{~S} 2}\right)$, where $v_{\mathrm{S} 1}=50 \mathrm{~km} \mathrm{~s}^{-1}$ and $v_{\mathrm{S} 2}=200 \mathrm{~km} \mathrm{~s}^{-1}$ are the lowest and highest shock velocities considered in this study.

value of $T_{\mathrm{d}} \sim 15-20 \mathrm{eV}$ seems appropriate for the perpendicular direction. The in-plane value, however, could be much higher, presumably above $30 \mathrm{eV}$.
Instead of graphite, fullerenes and carbon nanotubes may be a better analog for PAH molecules. For fullerene, $T_{\mathrm{d}}$ has been found between 7.6 and $15.7 \mathrm{eV}$ (Füller \& Banhart 1996). Single 
walled nanotubes consist of a cylindrically curved graphene layer. Unfortunately, also in this case the threshold for atomic displacement is not precisely determined. However it is expected to be lower than in a multi-layered tube, for which a value of $T_{\mathrm{d}} \sim 15-20 \mathrm{eV}$ has been found (Banhart 1997) close to the value of graphite. We note that 4.5 and $7.5 \mathrm{eV}$ are close to the energy of the single and double C-bond.

Because we cannot provide a well-defined $T_{0}$, we decided to explore a range of values, to study the impact of the threshold energy on the PAH processing. For our standard case, we adopt $7.5 \mathrm{eV}$ that we consider a reasonable value consistent with all the experimental data. However, we have varied $T_{0}$ from 4.5 to $15 \mathrm{eV}$.

Figure 3 shows the comparison between $S_{\mathrm{n}}, \sigma$ and $\langle T\rangle$ calculated for He on $\mathrm{C}$ assuming $T_{0}=4.5,7.5$ and $15 \mathrm{eV}$. Coherently with their definition, $S_{\mathrm{n}}$ and $\sigma$ increase with decreasing threshold, because more collisions are effective and the cross section increases with decreasing energy. Of course, the average energy transferred will decrease when the threshold energy is decreased.

In Sect. 4.1 we discuss the effect of the choice of different values for $T_{0}$ on the PAH survival in shocks.

\section{PAHs in shocks}

When grains and PAHs enter a shock they become charged and then gyrate around the compressed magnetic field lines. This leads to relative gas-particle velocities and hence to collisions with the gas (and other grains/PAHs). Collisions with the gas result in drag forces and therefore a decrease in the relative gasparticle velocity. However, these same collisions with the gas can also lead to the removal of atoms from the particle if the relative velocites are larger than the given threshold for an erosional process. The removal of carbon atoms from the PAH due to ion collisions, where the impact velocity is determined by the relative motion between the two partners, is the analog of the inertial sputtering of dust particles due to ion-grain collisions. In the following we will then refer to it using the term inertial, and the same will apply for all the related quantities.

In determining the processing of PAHs in shock waves, as with all grain processing, it is the relative gas-grain velocity profile through the shock that determines the level of processing. In calculating the relative ion-PAH velocity through the shock we use the same approach as in our previous work (Jones et al. 1994, 1996), which is based on the methods described in McKee et al. (1987). The PAH velocity is calculated using a 3D particle of the same mass as the 50 carbon atom PAH under consideration. The PAH velocity depends then on the PAH mass and average geometric cross section. For a PAH with $N_{\mathrm{C}}$ carbon atoms, these are given by $N_{\mathrm{C}} m_{\mathrm{C}}$ and $0.5 \pi a_{\mathrm{PAH}}^{2}$ with $a_{\mathrm{PAH}}$ given by $0.9 N_{\mathrm{C}} \AA$, appropriate for a compact PAH (Omont 1986) and the factor $1 / 2$ in the cross section takes the averaging over impact angle into account. The $\mathrm{PAH}$ and grain cross sections are very close (to within $11 \%$ for $N_{\mathrm{C}}=50$ ), thus we are justified in using the same numerical approach even though we are using a 3D grain to calculate the velocity profile of a 2D PAH through the shock.

The PAHs are injected into the shock with $3 / 4$ of the shock speed, as are all grains, and their trajectories are then calculated self-consistently with their coupling to the gas, until the relative gas-PAH veocity becomes zero. The velocity calculation includes the effects of the direct drag with the gas due to atom and ion collisions and the drag due to the ion-charged PAH interaction in the post-shock plasma. We find that for some shock

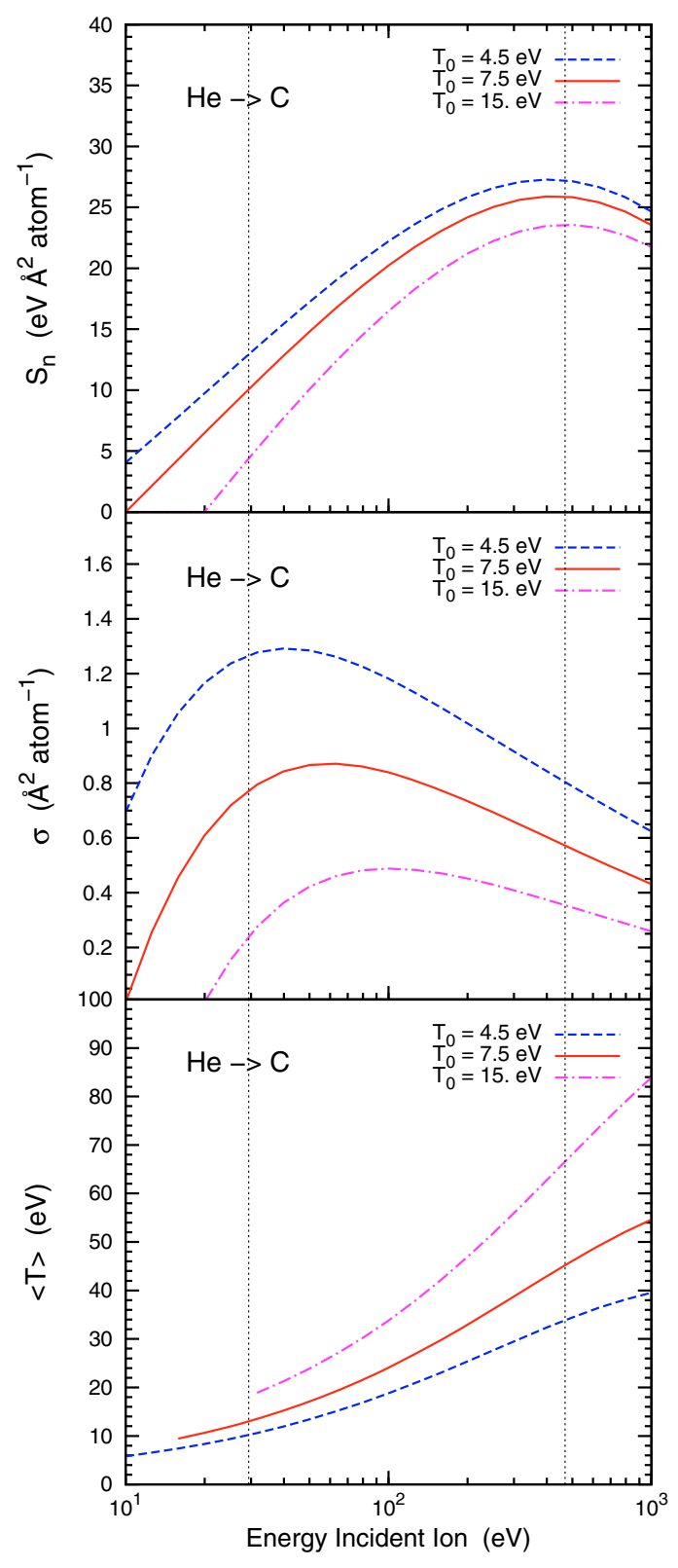

Fig. 3. The nuclear stopping cross section $S_{\mathrm{n}}(E)$, the cross section $\sigma(E)$ and the average energy transferred $\langle T(E)\rangle$ calculated for He ions impacting on a carbon atom, calculated for three values of the threshold energy $T_{0}: 4.5,7.5$ and $15 \mathrm{eV}$. The two vertical lines delimit the energy range of interest (cf. Fig. 2).

velocities, in our case for $v_{\mathrm{S}}=75$ and $100 \mathrm{~km} \mathrm{~s}^{-1}$, the PAHs (and grains) experience betatron acceleration in the post-shock gas. All the relevant expressions and assumptions for the calculation of the grain velocity, betatron acceleration and grain charge are fully described in McKee et al. (1987). Thus, in calculating the post-shock PAH velocity profiles, we follow exactly the same methods as used in our previous work. The structure of the $125 \mathrm{~km} \mathrm{~s}^{-1}$ shock is shown in Fig. 4 as a function of the column density $N_{\mathrm{H}}$. Figure 5 shows the velocity profile for a 50 carbon atom PAH in the same shock, together with the effective charge of the molecule, used to calculate the velocity profile itself. The $50 \mathrm{C}$-atoms PAH is positively charged (charge between +2 and +3 ) during the whole slowing process, and approaches neutrality at the end of the shock. 
E. R. Micelotta et al.: PAH processing in interstellar shocks

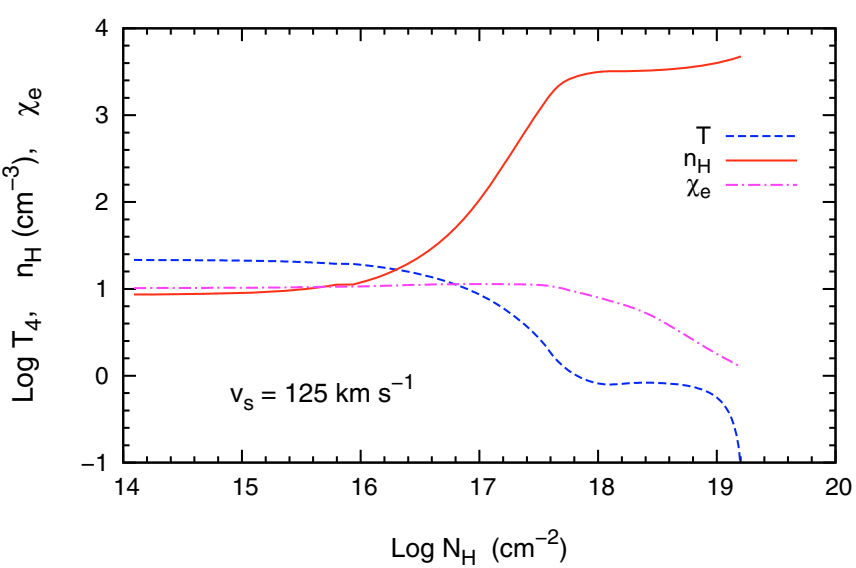

Fig. 4. The structure of the $125 \mathrm{~km} \mathrm{~s}^{-1}$ shock: temperature $T_{4}=$ $T / 10^{4} \mathrm{~K}$, hydrogen density $n_{\mathrm{H}}$ and electron relative abundance $\chi_{\mathrm{e}}$. All quantities are plotted as a function of the shocked column density $N_{\mathrm{H}}=n_{0} v_{\mathrm{S}} t$, where the preshock density $n_{0}=0.25 \mathrm{~cm}^{-3}$. To convert column density to time, use the following relation: $\log t(\mathrm{yr})=$ $\log N_{\mathrm{H}}\left(\mathrm{cm}^{-2}\right)-13.9$ (Jones et al. 1996).

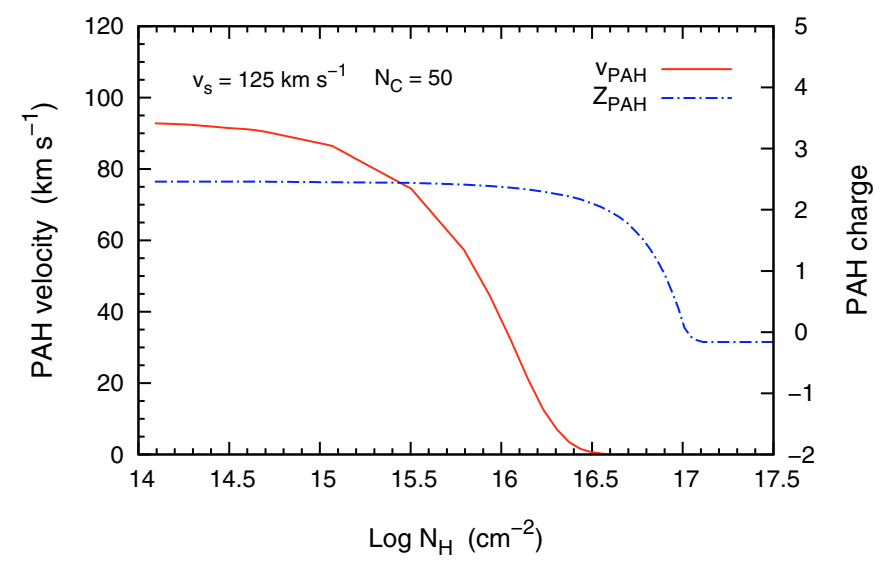

Fig. 5. The velocity profile of a $50 \mathrm{C}$-atom $\mathrm{PAH}$ in a shock with velocity $v_{\mathrm{S}}=125 \mathrm{~km} \mathrm{~s}^{-1}$. The PAH velocity $v_{\mathrm{PAH}}$, plotted as a function of the shocked column density $N_{\mathrm{H}}$, represents the relative velocity between the molecule and the ions present in the shock. Overlaid is the PAH charge $Z_{\mathrm{PAH}}$ along the shock, calculated using the theory described in McKee et al. (1987).

\subsection{Ion collisions: nuclear interaction}

Knowing the velocity profile of the $\mathrm{PAH}$, we can then calculate the inertial collision rate PAH-ions $R_{\mathrm{n}, \mathrm{I}}\left(\mathrm{s}^{-1}\right)$ through the shock. This is given by the following equation

$R_{\mathrm{n}, \mathrm{I}}\left(N_{\mathrm{H}}\right)=0.5 \chi_{i} n_{\mathrm{H}} v_{\mathrm{PAH}} \sigma N_{\mathrm{C}} F_{\mathrm{C}}$

where $n_{\mathrm{H}}\left(N_{\mathrm{H}}\right)$ is the hydrogen particle density along the shock, $v_{\mathrm{PAH}}\left(N_{\mathrm{H}}\right)$ the PAH-ion relative velocity along the shock and $\chi_{i}$ is the relative abundance of the projectile ion with respect to hydrogen. We adopt the gas phase abundances $\chi_{\mathrm{H}}: \chi_{\mathrm{He}}: \chi_{\mathrm{C}}=$ $1: 10^{-1}: 10^{-4}$, where the carbon abundance is between the values $(0.5-1) \times 10^{-4}$ and $1.4 \times 10^{-4}$ from Sofia (2009) and Cardelli et al. (1996) respectively.

The term $\sigma\left(N_{\mathrm{H}}\right)$ is the cross section averaged over those collisions that transfer an energy larger than the threshold energy $T_{0}$ per $\mathrm{C}$-atom and this cross section should therefore be multiplied by the number of carbon atoms in the PAH, $N_{\mathrm{C}}$. The factor 0.5 takes the angle averaged orientation into account (see Appendix C).

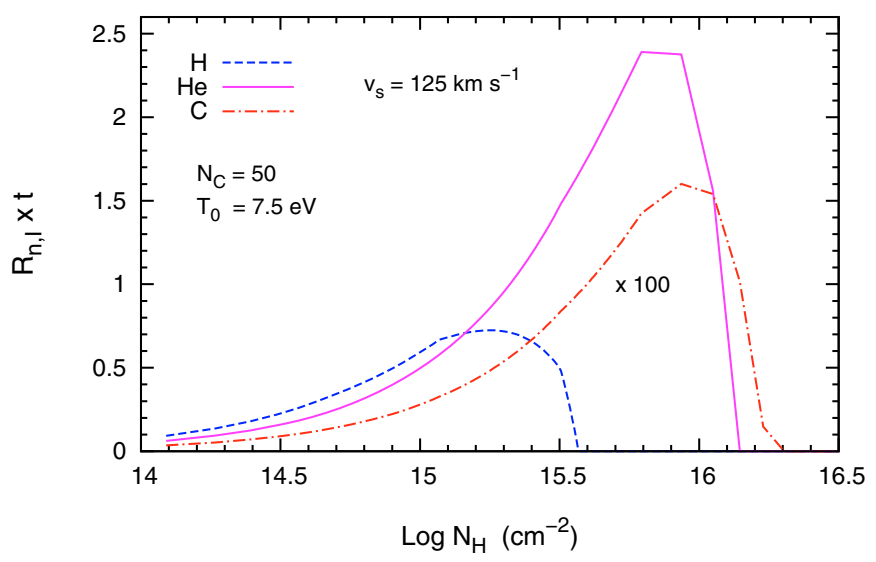

Fig. 6. The number of collisions $N_{\mathrm{t}}=R_{\mathrm{n}, \mathrm{I}} \times t$ of a 50 C-atom PAH with $\mathrm{H}, \mathrm{He}$ and $\mathrm{C}$ ions in a $125 \mathrm{~km} \mathrm{~s}^{-1}$ shock, as a function of the shocked column density, $N_{\mathrm{H}}$. The cross section has been evaluated for a threshold energy, $T_{0}$, of $7.5 \mathrm{eV}$. The carbon curve has been multiplyied by a factor 100 for comparison.

Because both collision partners are charged, the effect of the Coulombian potential must be included as well. Depending on whether the interaction is attractive or repulsive, the energy transfer cross section will be increased or reduced by the coulombian factor $F_{\mathrm{C}}$ given by

$F_{\mathrm{C}}=1-\frac{2 Z_{\mathrm{ion}} Z_{\mathrm{PAH}} e^{2}}{4 \pi \varepsilon_{0} a_{\mathrm{PAH}} M_{1} m_{\mathrm{H}} v_{\mathrm{PAH}}^{2}}$,

where $Z_{\text {ion }}=+1$ and $M_{1}$ are the charge and the atomic mass (in $\mathrm{amu}$ ) of the incident ion, $Z_{\mathrm{PAH}}$ is the charge of the PAHs along the shock, $a_{\mathrm{PAH}}$ is the $\mathrm{PAH}$ radius and $v_{\mathrm{PAH}}$ the $\mathrm{PAH}$-ion relative velocity. The constant $e$ is the electron charge, $\varepsilon_{0}$ is the permittivity of the free space and $m_{\mathrm{H}}$ the mass of the proton. The total number of destructive collisions is then given by the integral of the collision rate (Eq. (17)) behind the shock,

$N_{\mathrm{t}}=\int R_{\mathrm{n}, \mathrm{I}}\left(N_{\mathrm{H}}\right) \mathrm{d} t$

where it should be understood that the postshock column density $N_{\mathrm{H}}$ and the time, $t$, are related through $N_{\mathrm{H}}=n_{0} v_{\mathrm{S}} t$. With the proper cross section, this number $N_{\mathrm{t}}$, is then equal to the number of carbon atoms lost by a PAH in collisions with $\mathrm{H}, \mathrm{He}$, or $\mathrm{C}$.

Figure 6 illustrates the destructive collisions for a $50 \mathrm{C}$-atom PAH behind a $125 \mathrm{~km} \mathrm{~s}^{-1}$ shock assuming $T_{0}=7.5 \mathrm{eV}$. These results are plotted in such a way that equal areas under the curve indicate equal contributions to the total number of destructive collisions. $R_{\mathrm{n}, \mathrm{I}}$ drops precipitously because of the drop in relative PAH-gas velocity. Because heavier projectiles are more energetic in inertial collisions, this drop off shifts to higher column densities for heavier species. The results show that $\mathrm{He}$ is much more effective in destroying PAHs than $\mathrm{H}$ because of the increased energy transferred for heavier collision partners (cf. Fig. 2). The low abundance of $\mathrm{C}$ depresses its importance in inertial sputtering.

The number of carbon atoms in a PAH is now given by

$N_{\mathrm{C}}(t)=N_{\mathrm{C}}(0) \exp \left[-N_{\mathrm{t}} / N_{\mathrm{C}}\right]$

and the fraction of carbon atoms ejected from this PAH is

$F_{\mathrm{L}}=\left(1-\exp \left[-N_{\mathrm{t}} / N_{\mathrm{C}}\right]\right)$

where $N_{\mathrm{t}}$ is now evaluated throughout the shock. 
In the shocked gas, the velocity of the ions is not only determined by the relative motion with respect to the PAH (inertial case), but also by the temperature of the shocked gas. In principle, the inertial and thermal velocity should be added vectorally and averaged over the angle between the inertial motion and the (random) thermal motion as well as over the thermal velocity distribution. However, that becomes a quite cumbersome calculation and, hence, we will follow calculations for sputtering of dust grains in interstellar shocks (cf. Jones et al. 1994) and evaluate these two processes (inertial and thermal sputtering) independently. Studies have shown that this reproduces more extensive calculations satisfactorily (Guillet et al. 2007). The thermal destruction rate is given by

$R_{\mathrm{n}, T}\left(N_{\mathrm{H}}\right)=N_{\mathrm{C}} 0.5 \chi_{i} n_{\mathrm{H}} \int_{v_{0}}^{\infty} F_{\mathrm{C}}(v) v \sigma(v) f(v, T) \mathrm{d} v$

with $f(v, T)$ the Maxwellian velocity distribution. The temperature has to be evaluated along the shock profile (cf. Fig. 4) and care should be taken to only include velocities corresponding to energies larger than the threshold energy, $T_{0}$ (e.g., with $E>E_{0 n}$; cf., Table 2). The fraction of $\mathrm{C}$-atoms ejected by this process can be evaluated analogously to Eq. (21).

\subsection{Ion collisions: electronic interaction}

As reported in the introduction of the paper, the collision between PAH and ions triggers two simultaneous process, which can be treated separately: the nuclear stopping (elastic energy loss) and the electronic stopping (inelastic energy loss). The first has been extensively discussed in the previous sections, while for the full treatment of the electronic interaction we refer the reader to MJT. For the sake of clarity, we report here the essential concepts and the principal equations which will be used in the following.

The energy transferred to the electrons is spread out over the entire molecule, leaving the PAH in an excited state. Deexcitation occurs through two pricipal decay channels: emission of infrared photons and dissociation and loss of a $\mathrm{C}_{2}$ fragment. This latter is the process we are interested in, because it leads to the PAH fragmentation. The dissociation probability $p$ (see Sect. 4.1 in MJT) depends on the binding energy of the fragment $E_{0}$, on the PAH size, $N_{\mathrm{C}}$, and on the energy transferred, which in turns depends on the initial energy (velocity) of the projectile.

For a fixed value of the transferred energy, the dissociation probability decreases for increasing $E_{0}$ and $N_{\mathrm{C}}$ because either more energy is required in the bond that has to be broken or because the energy is spread over more vibrational modes and hence the internal excitation temperature is lower. On the other hand, the more energy that is deposited in the PAH, the higher is the dissociation probability. The energy transferred via electronic excitation increases with the energy of the projectile up to a maximum value, corresponding to an incident energy of $100 \mathrm{keV}$ for $\mathrm{H}$ (and higher for more massive particles), and decreases for higher energies. The deposited energy also increases with the path-length through the molecule and will be higher for larger PAHs impacted at grazing collision angles. For the shocks considered in this study, the energy transferred increases with incident energy (velocity) and hence the dissociation probability increases as well.
As for the nuclear stopping, also for the electronic interaction we have to consider the effect of both inertial and thermal velocities. The inertial collision rate is given by

$R_{\mathrm{e}, \mathrm{I}}\left(N_{\mathrm{H}}\right)=v_{\mathrm{PAH}} \chi_{i} n_{\mathrm{H}} F_{\mathrm{C}} \int_{\vartheta=0}^{\pi / 2} \sigma_{\mathrm{g}}(\vartheta) p\left(v_{\mathrm{PAH}}, \vartheta\right) \sin \vartheta \mathrm{d} \vartheta$

where $\vartheta$ is the angle between the axis normal to the PAH plane and the direction of the incoming ion. The term $\sigma_{\mathrm{g}}$ is the geometrical cross section seen by an incident particle with direction defined by $\vartheta$. The PAH is modelled as a thick disk with radius $a_{\mathrm{PAH}}$ and thickness $d$, then the cross section is given by

$\sigma_{\mathrm{g}}=\pi a_{\mathrm{PAH}}^{2} \cos \vartheta+2 a_{\mathrm{PAH}} d \sin \vartheta$

which reduces to $\sigma_{\mathrm{g}}=\pi a_{\mathrm{PAH}}^{2}$ for $\vartheta=0$ (face-on impact) and to $\sigma_{\mathrm{g}}=2 a_{\mathrm{PAH}} d$ for $\vartheta=\pi / 2$ (edge-on impact). The term $p\left(v_{\mathrm{PAH}}, \vartheta\right)$ represents the total probability for dissociation upon collision via electronic excitation, for a particle with relative velocity $v_{\mathrm{PAH}}$ and incoming direction $\vartheta$ (see Sect. 4.1 in MJT).

For the thermal collision rate we have

$R_{\mathrm{e}, T}\left(N_{\mathrm{H}}\right)=\int_{v_{0}}^{\infty} R_{\mathrm{e}, \mathrm{I}}(v) f(v, T) \mathrm{d} v$

where the temperature $T=T\left(N_{\mathrm{H}}\right)$ is evaluated along the shock. The lower integration limit $v_{0}$ is the ion velocity corresponding to $E_{0}$. The number of carbon atoms lost can be evaluated analogously to the nuclear interaction but care should be taken to include the loss of $2 \mathrm{C}$-atoms per collision.

There is a clear distinction between the nuclear and electronic interactions. In nuclear interactions, a C-atom is ejected because a direct collision with the impacting ion transfers enough energy and momentum to kick out the impactee instantaneously. In electronic interaction, the impacting ion excites the electrons of the PAH. Internal conversion transfers this energy to the vibrational motions of the atoms of the PAH. Rapid intramolecular vibrational relaxation leads then to a thermalization of this excess energy among all the vibrational modes and this can ultimately lead to dissociation (or relaxation through IR emission). The threshold energy in the nuclear process, $T_{0}$, differs therefore from the electronic dissociation energy, $E_{0}$. The latter really is a parameter describing the dissociation rate of a highly excited PAH molecules using an Arrhenius law and this does not necessarily reflect the actual binding energy of the fragment to the PAH species (cf. Tielens 2005). Following MJT, we will adopt the canonical value of $4.6 \mathrm{eV}$ for $E_{0}$. However, this energy is very uncertain and we will evaluate the effects of reducing and increasing this parameter to a value of 3.65 and $5.6 \mathrm{eV}$ respectively (MJT).

\subsection{Electron collisions}

For the full treatment of the PAH collisions with electrons, we refer again to the paper MJT, providing here a short summary of the basic concepts and equations.

Because of their small mass, the thermal velocity of the electrons always exceeds the inertial velocity of the PAH. Hence, only the thermal destruction needs to be evaluated. We follow the same formalism used for the electronic interaction in ionPAH collisions. The energy dumped into the molecule during collisions with electrons is spread over and determines (with $E_{0}$ and $N_{\mathrm{C}}$ ) the value of the dissociation probability. The electron energy loss rises sharply with the electron energy, reaching its maximum for incident energy around $100 \mathrm{eV}$. This energy range 
falls exactly in the interval relevant for our shocks, implying that the electrons optimally transfer their energy.

The thermal electron collision rate can be written as

$R_{\text {elec }, T}\left(N_{\mathrm{H}}\right)=\int_{v_{0, \text { elec }}}^{\infty} \Sigma(v) f(v, T) \mathrm{d} v$

$\Sigma(v)=v \chi_{\mathrm{e}} n_{\mathrm{H}} F_{\mathrm{Ce}} \int_{\vartheta=0}^{\pi / 2} \sigma_{\mathrm{g}}(\vartheta) p(v, \vartheta) \sin \vartheta \mathrm{d} \vartheta$

where $v_{0, \text { elec }}$ is the electron velocity corresponding to $E_{0}$ and $\chi_{\mathrm{e}}$ is the electron relative abundance along the shock. The electron coulombian factor $F_{\text {Ce }}$ is always equal to 1 (within less than $1 \%$ ) because electrons have low mass and high velocities with respect to ions. The temperature $T$ evaluated along the shock is the same as for the ions, but electrons will reach much larger velocities. From Eqs. (26) and (27) we expect then to find a significantly higher collision rate with respect to the ion case. The fraction of $\mathrm{C}$-atoms lost by electron collisions can be evaluated analogously to that for ions (cf. Eq. (21)).

\section{Results}

Figures 7 and 8 show the fraction of carbon atoms ejected from a 50 and $200 \mathrm{C}$-atoms $\mathrm{PAH}$ due to collisions with electrons and $\mathrm{H}, \mathrm{He}$ and $\mathrm{C}$, assuming the nuclear threshold energy $T_{0}=7.5 \mathrm{eV}$ and the fragment binding energy $E_{0}=4.58 \mathrm{eV}$. The results concerning nuclear, electronic and electron interaction are discussed in the following sections.

\subsection{PAH destruction via nuclear interactions}

For the inertial nuclear interactions, the fraction of ejected carbon atoms $F_{\mathrm{L}}$ depends on both $\sigma$ and $\chi$. Hydrogen has the highest abundance $\left(\chi_{\mathrm{H}}=1\right)$ but the lowest absolute value for the cross section (see Fig. 2). In addition, $\sigma$ is significantly different from zero only for the highest shock velocities. This results in contribution to atom ejection which is only relevant for $v_{\mathrm{S}}$ above $150 \mathrm{~km} \mathrm{~s}^{-1}$. Helium is ten times less abundant than hydrogen $\left(\chi_{\mathrm{He}}=0.1\right.$ ), but this is compensated for by a higher cross section for all shock velocities. In particular, the $\mathrm{C}$-atom ejection curve shows a peak between 50 and $125 \mathrm{~km} \mathrm{~s}^{-1}$ due to betatron acceleration: because of the higher velocity, the collision rate increases (cf. Eq. (17)) and then the PAHs experience more destructive collisions. After the peak, as expected the curve increases with the shock velocity. In the case of carbon, the increased cross section is not sufficient to compensate for the low abundance $\left(\chi_{\mathrm{C}}=10^{-4}\right)$, resulting in a totally negligible contribution to PAH destruction. For all shock velocities, the fraction of $\mathrm{C}$-atoms removed because of inertial nuclear interaction does not exceed the value of $20 \%$.

Concerning the thermal nuclear interaction, carbon does not contribute to PAH destruction because of its very low abundance compared to $\mathrm{H}$ and $\mathrm{He}$, as for the inertial case. For hydrogen and helium, as expected for low velocity shocks, the temperature is generally not sufficiently high to provide the ions with the energy required to remove $\mathrm{C}$-atoms. Nevertheless, the ions in the high velocity tail of the Maxwellian distribution can be energetic enough to cause $\mathrm{C}$-atom ejection, as can be seen for $\mathrm{He}$ at $100 \mathrm{~km} \mathrm{~s}^{-1}$. This is less evident for hydrogen. In this case the critical energy $E_{0 \mathrm{n}}$ is higher than for helium and carbon. The corresponding critical velocity $v_{0}$ will be higher as well. For the lower velocity shocks, the peak of the hydrogen maxwellian function $f(v, T)$ is well below $v_{0}$, as a consequence the integrand of Eq. (22) is close to zero over the integration range, and the same will be true for the collision rate. At the highest shock velocities the curves show a similar trend, with a steep rise beyond $125 \mathrm{~km} \mathrm{~s}^{-1}$ leading to complete PAH destruction, i.e. removal of ALL carbon atoms, for shock velocities above $150 \mathrm{~km} \mathrm{~s}^{-1}$. At around $135 \mathrm{~km} \mathrm{~s}^{-1}$ the hydrogen contribution becomes larger than that for helium. At these high velocities the $\mathrm{He}$ and $\mathrm{H}$ cross sections reach approximately their maximum values (cf. Fig. 2) and the abundance of $\mathrm{H}$ is a factor of 10 higher than for $\mathrm{He}$.

As discussed in Sect. 2.2.1, the threshold energy for carbon ejection via nuclear excitation is not well-constrained. We consider $T_{0}=7.5 \mathrm{eV}$ to be a reasonable value, but experimental determinations are necessary. Figure 9 illustrates how the fraction of ejected C-atoms changes as a function of the adopted value for the threshold energy. The curves show the cumulative effect of $\mathrm{H}$, He and $\mathrm{C}$, calculated for $T_{0}=4.5,7.5$ and $15 \mathrm{eV}$ in the inertial and thermal case. Both in the inertial and thermal case, the curves corresponding to the various thresholds follow the same trend, and for each value considered of $T_{0}$ the inertial destruction dominates at low velocity and the thermal destruction at high velocities. As expected the fraction of ejected $\mathrm{C}$-atoms increases for decreasing $T_{0}$ in the inertial case, while the curves shift to the left in the thermal case, implying that the PAHs will start to experience significant damage at lower shock velocities. Our results also show that, even assuming a high threshold energy, PAHs experience a substantial loss of carbon atoms, which is complete for velocities above $175 \mathrm{~km} \mathrm{~s}^{-1}$ is all cases.

Finally, we investigated how the nuclear destruction process depends on the size of the PAH. Figure 8 shows the fraction of ejected carbon atoms from a big PAH with $N_{\mathrm{C}}=200$. The destruction of a $200 \mathrm{C}$-atom PAH follows the same trends with shock velocity as for the $50 \mathrm{C}$-atom case and the curves are almost identical. This is due to the fact that the velocity and temperature profiles for the 50 and $200 \mathrm{C}$-atoms molecules are quite similar, and the collision rate and $F_{\mathrm{L}}$ scale linearly with $N_{\mathrm{C}}$ in both the inertial and thermal case (see Eqs. (17), (21) and (22)).

\subsection{PAH destruction via electronic interaction by ion collisions}

Inspection of Figs. 7 and 8 reveals that electronic excitation by impacting ions plays only a marginal role in the destruction process. For both PAH sizes, carbon is unimportant because of its very low abundance. In the inertial case $\mathrm{H}$ does not contribute and $\mathrm{He}$ contributes marginally at the highest shock velocities, while in the thermal case they lead to a substantial atomic loss only for $N_{\mathrm{C}}=50$ in the highest velocity shock $\left(200 \mathrm{~km} \mathrm{~s}^{-1}\right)$. For a $50 \mathrm{C}$-atom $\mathrm{PAH}$, the low destruction rate due to electronic excitation reflects the small cross section for this process for these low velocity shocks. The inertial velocities of the PAH lead to electronic excitation only being important for the highest shock velocities where the impacting ions have a high enough temperature to excite the PAHs sufficiently (cf. MJT). The larger number of modes available in $200 \mathrm{C}$-atom PAHs, makes the electronic excitation of such PAHs completely negligible over the full velocity range of the shocks considered here.

\subsection{PAH destruction due to electron collisions}

The fractional carbon atom loss $F_{\mathrm{L}}$ due to collisions with thermal electrons is also shown in Figs. 7 and 8. For $N_{\mathrm{C}}=50$, the number of ejected carbon atoms rises sharply above $75 \mathrm{~km} \mathrm{~s}^{-1}$, leading to total destruction above $100 \mathrm{~km} \mathrm{~s}^{-1}$. For $N_{\mathrm{C}}=200$, the 
A\&A 510, A36 (2010)
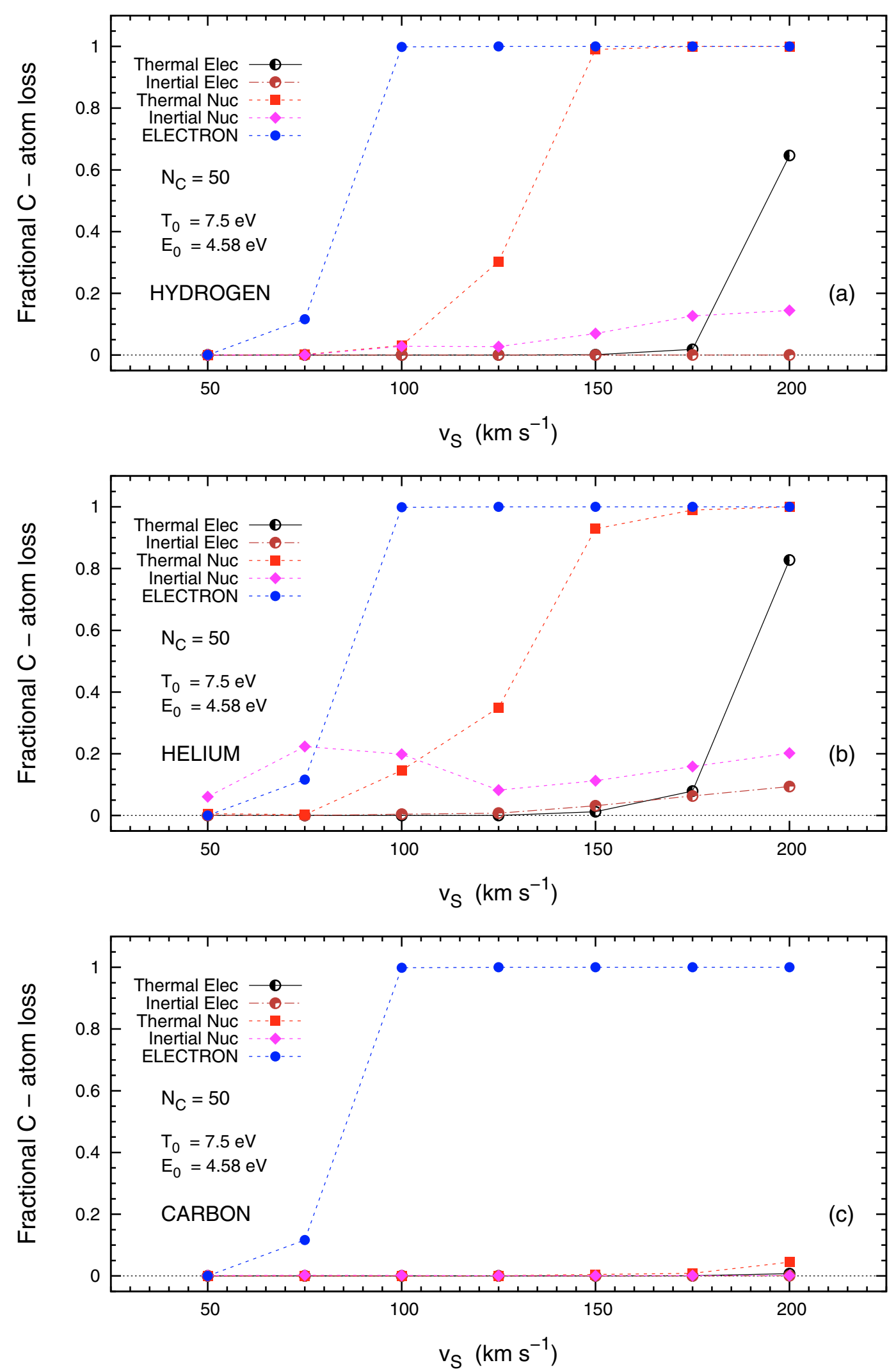

Fig. 7. The fractional $\mathrm{C}$-atom loss $F_{\mathrm{L}}$, due to collisions with $\mathrm{H}, \mathrm{He}$ and $\mathrm{C}$ ions and electrons, as a function of the shock velocity. $F_{\mathrm{L}}$ is defined as the total number of ejected carbon atoms divided by the initial number $N_{\mathrm{C}}$ of $\mathrm{C}$-atoms in the PAH molecule. The destructive effect of thermal and inertial "sputtering" induced by collisions with the projectiles are shown. In the nuclear case, each lost atom is the result of a single collision with a given projectile, in other words, every collision removes a $\mathrm{C}$-atom from the molecule so, the number of destructive collisions equals the number of ejected carbon atoms. For electrons as projectiles and for electronic interaction, each collision leads to the ejection of two carbon atoms. The points are calculated for a $50 \mathrm{C}$-atoms PAH assuming the nuclear threshold energy $T_{0}=7.5 \mathrm{eV}$ and the electronic dissociation energy $E_{0}=4.58 \mathrm{eV}$. 
E. R. Micelotta et al.: PAH processing in interstellar shocks
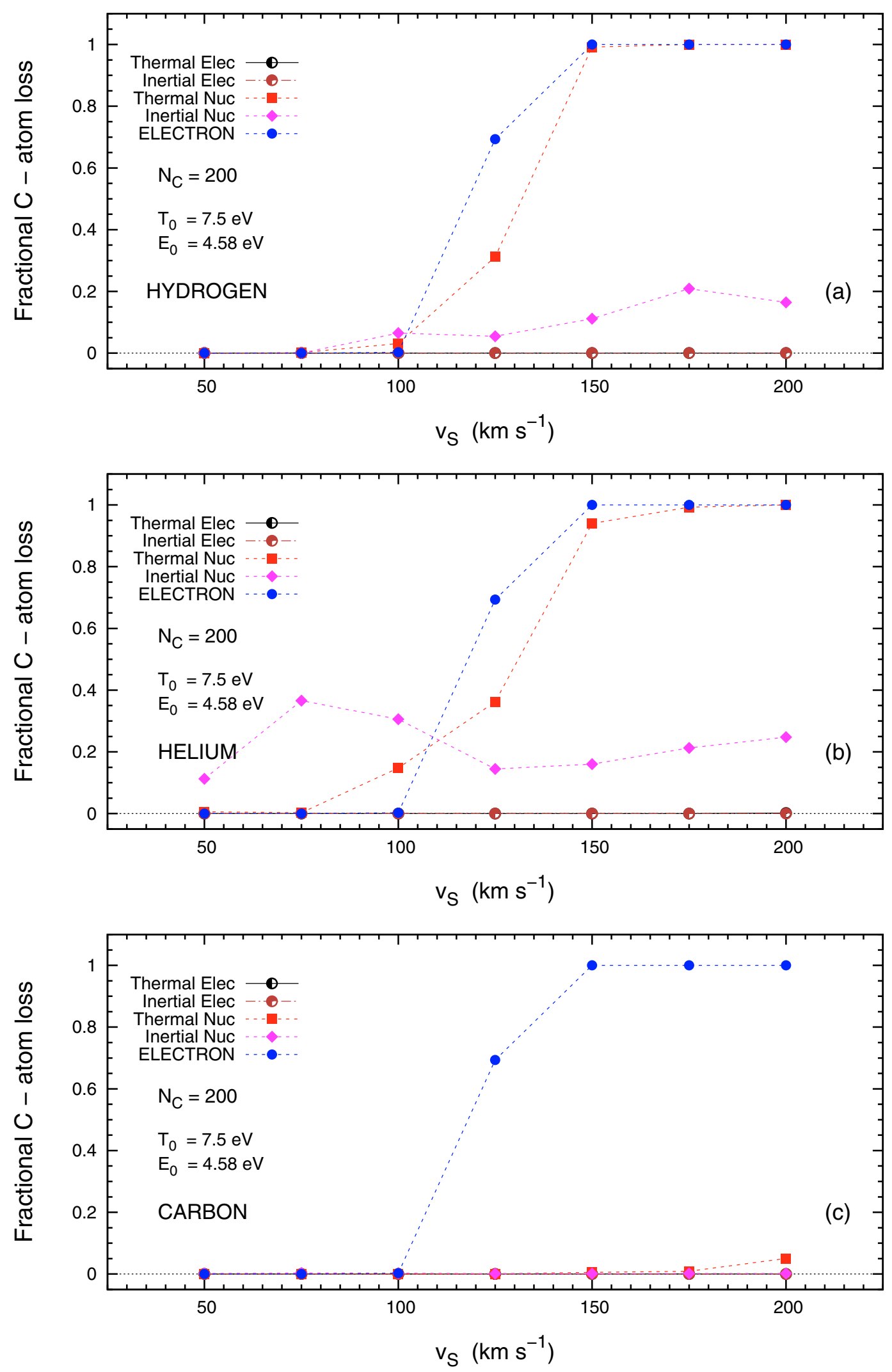

Fig. 8. Same as Fig. 7 calculated for a 200 carbon atoms PAH.

damage is negligible up to $100 \mathrm{~km} \mathrm{~s}^{-1}$, increases significantly beyond that and leads to complete destruction above $150 \mathrm{~km} \mathrm{~s}^{-1}$.

The energy transferred by impacting electrons rises sharply for velocities in excess of $2 \times 10^{3} \mathrm{~km} \mathrm{~s}^{-1}$. This results in a dissociation probability $p$ shaped as a step function: for $v \gtrsim 2 \times$ $10^{3} \mathrm{~km} \mathrm{~s}^{-1} p$ jumps from values close to zero up to 1 . This limiting velocity applies to a $50 \mathrm{C}$-atoms $\mathrm{PAH}$; for $N_{\mathrm{C}}=200$ the value is higher $\left(4 \times 10^{3} \mathrm{~km} \mathrm{~s}^{-1}\right)$, due to the fact that for a bigger PAH 


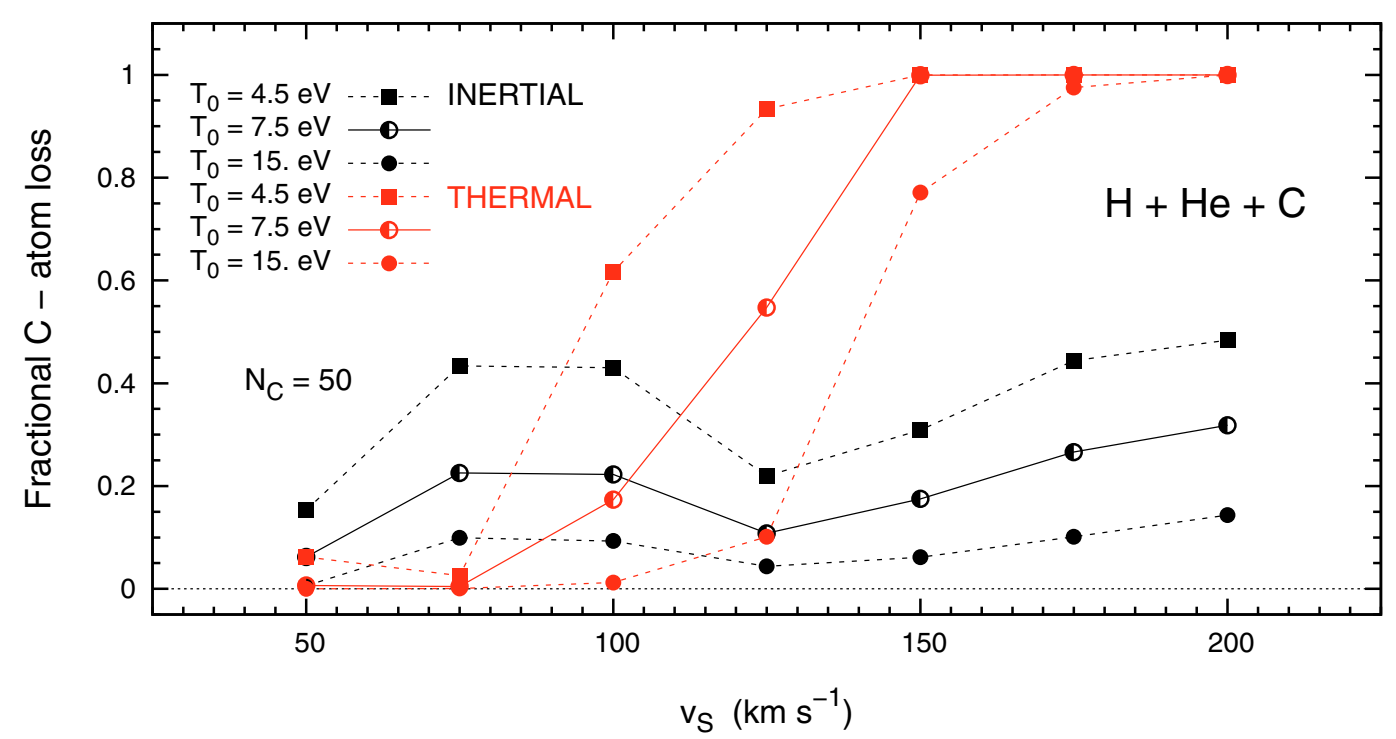

Fig. 9. Carbon atom loss due to collisions with thermal and inertial ions $(\mathrm{H}+\mathrm{He}+\mathrm{C})$ via nuclear interaction. The figure shows the comparison betwen the three threshold values $T_{0}=4.5,7.5$ and $15 \mathrm{eV}$ for a 50 carbon atom PAH.

more energy has to be transferred for dissociation. These velocities correspond to electron temperatures of $10^{5} \mathrm{~K}$ and $3 \times 10^{5} \mathrm{~K}$, which are reached for shock velocities of approximately 100 and $150 \mathrm{~km} \mathrm{~s}^{-1}$, respectively.

\subsection{Summary}

A summary of our findings from Figs. 7 and 8 is presented in Fig. 10. This shows the fractional atomic loss, $F_{\mathrm{L}}$, due to electron and ion collisions, calculated for the two PAH sizes $N_{\mathrm{C}}=$ 50 and 200. To show how the fractional loss changes as a function of the adopted value for $E_{0}$, we added the results obtained assuming for the electronic dissociation energy the values 3.65 and $5.6 \mathrm{eV}$, lower and higher respectively than our standard value $4.58 \mathrm{eV}$.

For ionic collisions, $F_{\mathrm{L}}$ is determined by nuclear interaction: inertial for low $v_{\mathrm{S}}$ and thermal for high $v_{\mathrm{S}}$. For the $50 \mathrm{C}$-atoms $\mathrm{PAH}$, the electronic contribution emerges for the lowest value of $E_{0}, 3.65 \mathrm{eV}$. As already mentioned, the dissociation probability increases for decreasing $E_{0}$, so we are not surprised to find $F_{\mathrm{L}}$ enhanced by electronic excitation (both inertial and thermal). The electronic contribution desappears for higher dissociation energies, as demonstrated by the coincidence between the curves for $E_{0}=4.58$ and $5.6 \mathrm{eV}$. For $N_{\mathrm{C}}=200$, all three ionic curves are coincident, indicating that electronic excitation by impacting ions does not contribute to PAH destruction below $150 \mathrm{~km} \mathrm{~s}^{-1}$. Above this value, the carbon loss due to electronic interaction is covered by the other processes.

The shift between the ionic curves for the two PAH sizes is due to the small differences in the velocity profiles - due to betatron acceleration -, which imply in the inertial case a slightly higher damage for the bigger PAH. In the thermal case $F_{\mathrm{L}}$ is instead independent on the PAH size because the number of ejected C-atoms scales linearly with $N_{\mathrm{C}}$ (see Sect. 4.1). The resulting effect is an almost linear rise for both PAH sizes up to $150 \mathrm{~km} \mathrm{~s}^{-1}$, beyond which the destruction is complete.

The "Electron" curves reproduce the behaviour observed in Figs. 7 and 8 . The comparison with the ionic $F_{\mathrm{L}}$ clearly indicates that a 50 carbon atoms PAH is already damaged in a non-negligible way in low velocity shocks $\left(50-75 \mathrm{~km} \mathrm{~s}^{-1}\right.$, ion collisions) and is totally destroyed above $100 \mathrm{~km} \mathrm{~s}^{-1}$ (electron collisions). When $E_{0}=3.65 \mathrm{eV}$, the fraction of ejected C-atom for $v_{\mathrm{S}}=75 \mathrm{~km} \mathrm{~s}^{-1}$ increases from 0.1 to $\sim 0.35$ but the minimum shock velocity for complete destruction is unchanged $\left(100 \mathrm{~km} \mathrm{~s}^{-1}\right)$. For $v_{\mathrm{S}} \geq 75 \mathrm{~km} \mathrm{~s}^{-1}$, the $5.6 \mathrm{eV}$ curve is almost parallel to the $3.65 \mathrm{eV}$ curve and shifted by $25 \mathrm{~km} \mathrm{~s}^{-1}$ toward higher shock velocities. For the $200 \mathrm{C}$-atom PAH, the carbon atom loss is dominated by ionic collisions for shocks with velocity below $100 \mathrm{~km} \mathrm{~s}^{-1}$. Above this value, the combined effect of ions and electrons leads to a complete destruction. A lower electronic dissociation energy shifts back by $25 \mathrm{~km} \mathrm{~s}^{-1}$ the minimum shock velocity required for total carbon ejection. When $E_{0}=5.6 \mathrm{eV}$, destruction starts to be important only above $125 \mathrm{~km} \mathrm{~s}^{-1}$, and becomes almost complete at $150 \mathrm{~km} \mathrm{~s}^{-1}$.

We adopt the quantity $F_{\mathrm{L}}$ as destruction efficiency to calculate the PAH lifetime in shocks.

\subsection{Uncertainties discussion}

The main sources of uncertainties which have to be considered for this study are related to the adopted shock profiles, to the accuracy of the fitting function for the ZBL nuclear stopping cross section, and to the choice of an appropriate value for the nuclear threshold energy $T_{0}$ and for the electronic dissociation energy $E_{0}$.

The uncertainties related to the adopted shock profiles here are principally due to our assumption that we can equivalently treat a small, two-dimensional PAH molecule as a small threedimensional grain. In the calculation of the PAH velocity profiles through the shocks we use the same formalism as for the grains (Jones et al. 1996), i.e., we assume that the PAH behaves as a three-dimensional grain of the same mass. Any uncertainties are then due to the inherent differences in the cross section to mass ratios for PAHs and grains. As mentioned in Sect. 3, once the PAH cross section is averaged over all possible orientations, the differences in the PAH and grain cross sections turn out to be only of the order of $11 \%$, for a 50 carbon atom PAH, and are therefore rather small compared to the other uncertainties that we discuss here. 
E. R. Micelotta et al.: PAH processing in interstellar shocks
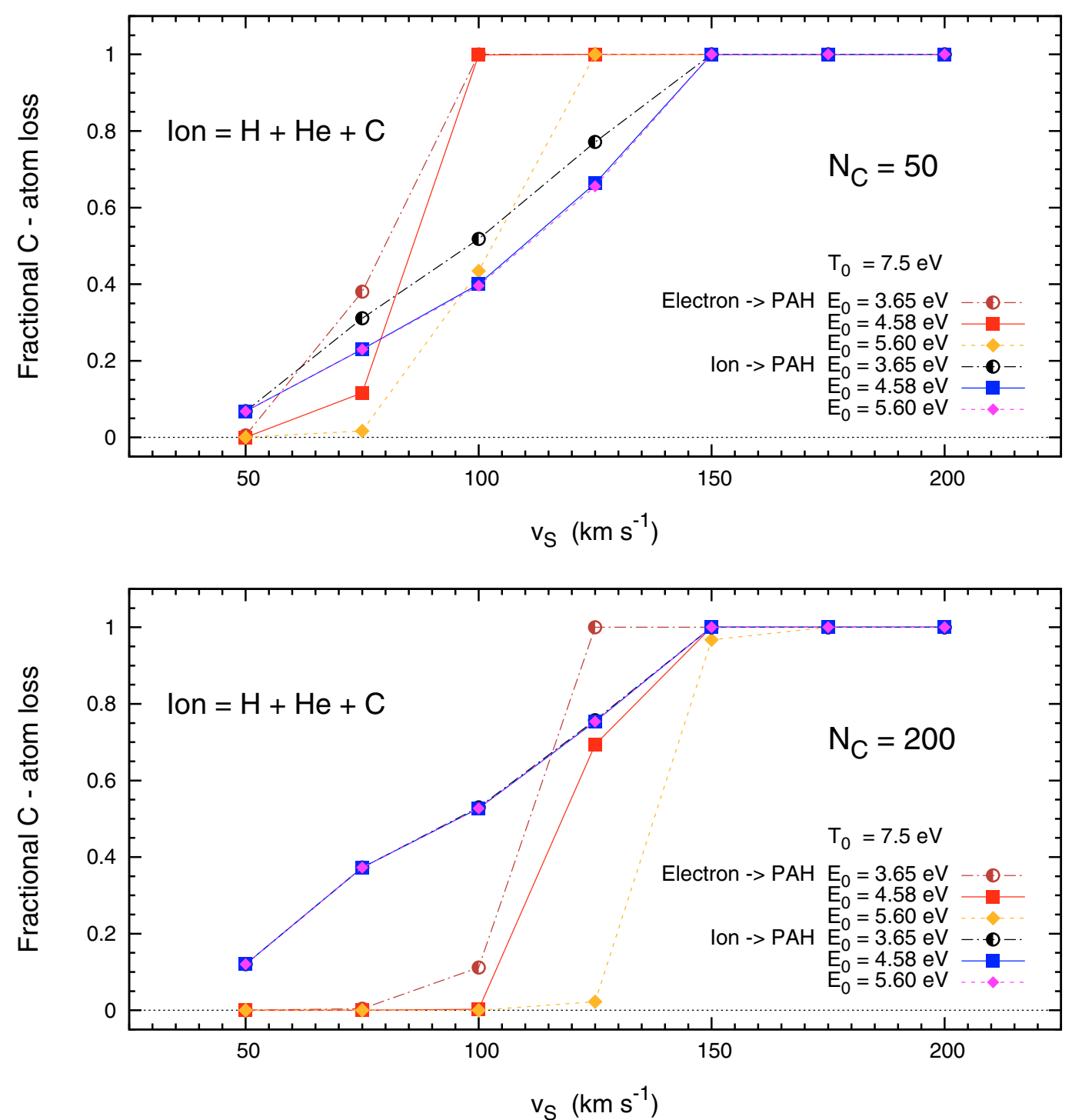

Fig. 10. The fractional C-atom loss $F_{\mathrm{L}}$ as a function of the shock velocity, calculated for three values of the parameter $E_{0}: 3.65,4.58$ and $5.6 \mathrm{eV}$. $F_{\mathrm{L}}$ is defined as the total number of ejected carbon atoms divided by the initial number $N_{\mathrm{C}}$ of C-atoms in the PAH molecule. For the two PAH sizes $N_{\mathrm{C}}=50$ (top panel) and $N_{\mathrm{C}}=200$ (bottom panel), the curves labelled 'Ion' illustrate the cumulative effect of all destructive processes for the ions considered in this study: inertial and thermal "sputtering" due to nuclear and electronic excitation during PAH collisions with $\mathrm{H}, \mathrm{He}$ and $\mathrm{C}$ in the shocks. The curves labelled "Electron" show the destructive effect of thermal electrons. We assume $T_{0}=7.5 \mathrm{eV}$.

The accuracy of the ZBL nuclear stopping cross section depends on the accuracy of the single analytical function used by Ziegler et al. (1985) to calculate the interatomic potentials between atoms. This universal function has been compared with experimentally determined potentials, with a resulting standard deviation between theory and experiment of 5\% (O'Connor \& Biersack 1986). An additional test has been made comparing the results from the ZBL function with much more complex theoretical calculations including more effects. In this case as well the results agree within few percent (see Ziegler et al. 1985, and references therein).

The main source of uncertainty in the nuclear stopping calculation is the choice of the threshold energy $T_{0}$. This quantity is not well constrained (see Sect. 2.2.1) so we explored a set of plausible values. In Fig. 9 we plotted together the values for $F_{\mathrm{L}}$ resulting from the total effect of $\mathrm{H}, \mathrm{He}$ and $\mathrm{C}$, calculated in both inertial and thermal case for three different threshold energies $T_{0}=4.5,7.5$ and $15 \mathrm{eV}$. The curves corresponding to the highest and lowest threshold $T_{0}^{\max }$ and $T_{0}^{\min }$ identify a region which can be interpreted as the variation in the amount of destruction due to the uncertainty in the threshold energy. For the inertial case, this uncertainty introduces a variation in the destruction efficiency of a factor less than about 2. For the thermal case, the uncertainty introduces a shift of the critical shock velocity above which thermal destruction is dominant from about 100 to $150 \mathrm{~km} \mathrm{~s}^{-1}$ for $T_{0}$ ranging from 4.5 to $15 \mathrm{eV}$.

An important issue for the electronic and electron stopping calculation is the choice of the value for the parameter $E_{0}$. We adopt the value $4.58 \mathrm{eV}$, which has been extrapolated for interstellar conditions from experimental data. Unfortunately the extrapolation procedure is very model-dependent, so the same set of experimental data can lead to significantly different values for the interstellar $E_{0}$. The problematic fragment binding energy is extensively discussed in MJT, the result of a different choice for $E_{0}(3.65,4.58$ and $5.6 \mathrm{eV})$ on PAH processing by shocks is shown in Fig. 10. The differences are quite significant, indicating the importance of experimental studies on the critical energy $E_{0}$ describing the dissociation probability of highly excited PAHs. 
Table 3. PAH destruction analytical fit parameters and survival timescales for electron and ion collisions.

\begin{tabular}{|c|c|c|c|c|c|c|}
\hline & \multicolumn{2}{|c|}{ Electron } & \multicolumn{2}{|c|}{ Ion } & \multirow{2}{*}{\multicolumn{2}{|c|}{$\begin{array}{c}\text { Fit } \\
\text { parameters }\end{array}$}} \\
\hline & \multirow{2}{*}{$\frac{N_{\mathrm{C}}=50}{0.50<v_{\mathrm{S} 7}<0.75}$} & \multirow{2}{*}{$N_{\mathrm{C}}=200$} & $N_{\mathrm{C}}=50$ & $N_{\mathrm{C}}=200$ & & \\
\hline $\bar{\varepsilon}\left(v_{\mathrm{S} 7}\right)=m_{1} x+q_{1}$ & & & - & - & $m_{1}=0.464$ & $q_{1}=-0.232$ \\
\hline$\varepsilon\left(v_{\mathrm{S} 7}\right)=m_{2} x+q_{2}$ & $0.75 \leq v_{\mathrm{S} 7} \leq 1.00$ & - & - & - & $m_{2}=3.536$ & $q_{2}=-2.536$ \\
\hline$\varepsilon\left(v_{\mathrm{S} 7}\right)=m_{3} x+q_{3}$ & - & - & - & $0.50 \leq v_{\mathrm{S} 7} \leq 1.50$ & $m_{3}=0.879$ & $q_{3}=-0.319$ \\
\hline$\varepsilon\left(v_{\mathrm{S} 7}\right)=a x^{b}$ & - & - & $0.50 \leq v_{\mathrm{S} 7} \leq 1.50$ & - & $a=0.405$ & $b=2.232$ \\
\hline$\varepsilon\left(v_{\mathrm{S} 7}\right)=c \ln \left(x^{d}\right) / x$ & - & $0.50 \leq v_{\mathrm{S} 7} \leq 1.50$ & - & - & $c=5.366$ & $d=0.7$ \\
\hline$\varepsilon\left(v_{\mathrm{S} 7}\right)=k$ & $1.00 \leq v_{\mathrm{S} 7} \leq 2.00$ & $1.50 \leq v_{\mathrm{S} 7} \leq 2.00$ & $1.50 \leq v_{\mathrm{S} 7} \leq 2.00$ & $1.50 \leq v_{\mathrm{S} 7} \leq 2.00$ & & $=1$ \\
\hline$t_{\mathrm{SNR}}(\mathrm{yr})$ & $1.6 \times 10^{8}$ & $4.0 \times 10^{8}$ & $1.8 \times 10^{8}$ & $1.4 \times 10^{8}$ & & \\
\hline
\end{tabular}

Notes. Fit parameters and survival timescales calculated assuming $T_{0}=7.5 \mathrm{eV}$ and $E_{0}=4.6 \mathrm{eV}$.

To summarize, the errors related to the shock profiles and ZBL fitting function are quite small, for $T_{0}$ we identified a range of plausible values, but experimental determinations would be desirable, while the choice of the parameter $E_{0}$ is very uncertain and urgently requires a better determination.

\section{Discussion}

\subsection{PAH lifetime in shocks}

To calculate the timescale for supernova shock waves to destroy the interstellar PAHs in the Galaxy, $t_{\mathrm{SNR}}$, we adopt the same approach used in our previous works (Jones et al. 1994, 1996), which is based on the method of McKee (1989):

$t_{\mathrm{SNR}}=\frac{M_{\mathrm{ISM}}}{\left(1 / \tau_{\mathrm{SN}}^{\prime}\right) \int \varepsilon\left(v_{\mathrm{S}}\right) \mathrm{d} M_{\mathrm{S}}\left(v_{\mathrm{S}}\right)}$

where $M_{\text {ISM }}=4.5 \times 10^{9} M_{\odot}$ is the mass of the Galactic interstellar medium (gas and dust including PAHs), $\tau_{\mathrm{SN}}^{\prime}=125 \mathrm{yr}$ is the effective interval between supernovae (McKee 1989), $\varepsilon\left(v_{\mathrm{S}}\right)$ is in this case the efficiency of PAH destruction by a shock of velocity $v_{\mathrm{S}}$ and $M_{\mathrm{S}}$ is the mass of gas shocked to at least $v_{\mathrm{S}}$ by a supernova remnant in the Sedov-Taylor stage (in this stage the energy is conserved, so that $M_{\mathrm{S}} v_{\mathrm{S}}^{2} \propto E$ ). In a three-phase model of the interstellar medium (McKee \& Ostriker 1977), with a ratio between warm and hot intercloud filling factor of $f_{\mathrm{w}} / f_{\mathrm{h}}=$ $0.3 / 0.7=0.43$, the mass $M_{\mathrm{S}}$ and the timescale $t_{\mathrm{SNR}}$ become

$M_{\mathrm{S}}\left(v_{\mathrm{S}}\right)=2914 M_{\odot} / v_{\mathrm{S} 7}^{2}$

$t_{\mathrm{SNR}}=\frac{9.7 \times 10^{7}}{\int \varepsilon\left(v_{\mathrm{S} 7}\right) / v_{\mathrm{S} 7}^{3} \mathrm{~d} v_{\mathrm{S} 7}} \mathrm{yr}$

were $v_{\mathrm{S} 7}$ is shock velocity in units of $100 \mathrm{~km} \mathrm{~s}^{-1}$ and the assumed mean supernova energy is $10^{51} \mathrm{erg}$.

Using our calculated fractional destruction data, we derived analytical expressions for the destruction efficiency $\varepsilon\left(v_{\mathrm{S} 7}\right)$ for electrons and ions. The ionic term represents the total contribution of all considered ions $(\mathrm{H}, \mathrm{He}$ and $\mathrm{C}$ ) and processes (nuclear and electronic stopping, inertial and thermal). Below $150 \mathrm{~km} \mathrm{~s}^{-1}$, we adopt a power law fit for $N_{\mathrm{C}}=50$ and a linear fit for $N_{\mathrm{C}}=200$, while for the remaining velocities the efficiency is 1 . For electrons, for $F_{\mathrm{L}}<1$, the destruction efficiencies are well fit by two linear functions $\left(N_{\mathrm{C}}=50\right)$ and a logarithmic function $\left(N_{\mathrm{C}}=200\right)$. The analytical fits reproduce the calculated data within few percent. The functional form, fitting parameters and corresponding timescales calculated from Eq. (30) are reported in Table 3.
For electron collisions we find $t_{\mathrm{SNR}}=1.6 \times 10^{8} \mathrm{yr}$ for $N_{\mathrm{C}}=50$ and $4.0 \times 10^{8} \mathrm{yr}$ for $N_{\mathrm{C}}=200$. In case of ion collisions the lifetimes are $1.8 \times 10^{8} \mathrm{yr}$ and $1.4 \times 10^{8} \mathrm{yr}$ for the small and big molecule respectively. The largest uncertainty in these lifetimes result from the uncertainty in the values adopted for $T_{0}$ and $E_{0}$ (cf. Sect. 4.5). If we assume the lowest values considered ( 4.5 and $3.65 \mathrm{eV}$ ), these lifetimes decrease to $9.2 \times$ $10^{7} \mathrm{yr}$ and $8 \times 10^{7} \mathrm{yr}$ (for $N_{\mathrm{C}}=50$ and 200 respectively), while the maximum values considered for these energies result in lifetimes of $2.5 \times 10^{8} \mathrm{yr}$ and $3.3 \times 10^{8} \mathrm{yr}$ for the small and big PAH. Thus, while these parameters are quite uncertain, the derived values for the lifetimes are quite robust. Essentially, PAHs are destroyed by shocks larger than about $100 \mathrm{~km} \mathrm{~s}^{-1}$ and, typically, interstellar gas encounters such shocks once every 100 million years (McKee 1989).

From the results obtained assuming our standard values $\left(T_{0}=7.5 \mathrm{eV}\right.$ and $\left.E_{0}=4.6 \mathrm{eV}\right)$, we argue then that small PAHs are preferentially destroyed by electrons whereas big PAHs are more affected by ions.

Our derived values for $t_{\mathrm{SNR}}$ for PAHs are significantly shorter than the $6 \times 10^{8}$ yr calculated by Jones et al. (1996) for graphite/amorphous carbon grains in the warm intercloud medium. Ignoring betatron acceleration, the total number of collisions per $\mathrm{C}$-atom required for stopping the inertial motion is independent of grain size. The difference in lifetimes reflects then a difference in sputtering efficiency. This is not surprising because of the different approaches adopted for the ion-particle interactions for PAHs and grains. In both cases, and for the shocks that we consider here, it is the sputtering processes that completely dominate dust destruction. In the case of grains not every atom that is "knocked on" by an incident ion is lost, i.e. sputtered, from the grain. The displaced atom is often embedded deeper into the grain and therefore not sputtered from the grain even when the displacement energy significantly exceeds the threshold energy for target atom displacement. This is reflected in the fact that, for grains, the sputtering yield is usually much less that unity. The shorter lifetime for the PAHs is then be ascribed to the fact that in any incident ion interaction the target atom is always lost from the PAH when the energy to displace it is greater that the required threshold energy. In this case the equivalent PAH sputtering yield is then unity.

Our derived PAH lifetime is much closer to the value of $\sim 2 \times 10^{8}$ yr found for a size distribution of hydrogenated amorphous carbon (a-C:H) grains, typical of the diffuse interstellar medium, by Serra Díaz-Cano \& Jones (2008). This is perhaps just coincidental; it has its origin in lower average binding energy of $\mathrm{C}$-atoms in amorphous carbon than in graphite. a$\mathrm{C}: \mathrm{H}$ grains are found, as we find for PAHs, to be more susceptible to sputtering erosion than graphite/amorphous carbon grains 
(Jones et al. 1996). However PAHs are much more susceptible to erosion than a-C:H in fast shocks $\left(\geq 150 \mathrm{~km} \mathrm{~s}^{-1}\right)$ and this is simply due to their small sizes. Thermal sputtering in the hot post-shock gas of fast shocks is proportional to the surface area and, small particles having a larger surface area per unit mass than large particles, are more rapidly eroded.

\subsection{Astrophysical implications}

As shown in Sect. 5.1, the PAH lifetime against shock destruction is much shorter than the stardust injection timescale into the interstellar medium $t_{\text {inj }}=2.5 \times 10^{9} \mathrm{yr}$. Gas shocked to velocities of the order of $50-150 \mathrm{~km} \mathrm{~s}^{-1}$ is observed in many regions of the interstellar medium: e.g. toward the star $\zeta$ Ori (Welty et al. 2002), in Herbig-Haro jets in the Orion and Vela star forming regions (Podio et al. 2006), and in the local interstellar cloud (Slavin 2008). Hence, according to our calculations, PAH destruction should be widespread in the ISM.

If we assume globally that the same $t_{\text {inj }}$ holds for PAHs as for "standard" interstellar dust, i.e., that dust and PAH formation are coeval in and around evolved stars, our calculated survival times for PAHs, i.e., $t_{\mathrm{SNR}}$, indicate that they need to be re-formed in the ISM even more rapidly than the larger interstellar grains. If PAHs are formed by the fragmentation of larger carbonaceous grains then their "effective" survival time must just be the same as that of the larger grains from which they originate. However, this can only be true for low velocity shocks, or turbulent regions of the ISM, where grain-grain collisions at relatively low velocities (of the order of a few $\mathrm{km} \mathrm{s}^{-1}$ ) can form PAHs via fragmentation and where there is no associated destructive process in operation.

On a region by region basis our results indicate that PAHs should not exist in environments shocked to high velocities $\left(>100 \mathrm{~km} \mathrm{~s}^{-1}\right)$. We conclude that PAHs that exist in unshocked regions do not survive the passage of shocks with velocities above 100-150 $\mathrm{km} \mathrm{s}^{-1}$ (depending on their size). They are in fact destroyed rather early in in the shock at shocked column densities of the order of $10^{16}-10^{17} \mathrm{~cm}^{-2}$. Any "daughter" PAHs produced in the post-shock region, by grain fragmentation in grain-grain collisions at shocked column densities of the order of $10^{17}-10^{18} \mathrm{~cm}^{-2}$, will be destroyed by erosion due to their high injection velocities into the gas following the fragmentation of their larger, "parent" grains that undergo betatron-acceleration (Jones et al. 1996). Thus, high velocity shocks destroy all the PAHs that they both interact with and produce by fragmentation in high velocity grain-grain collisions.

In contrast observations show that PAHs lock up about $3 \%$ of the elemental carbon in the ISM (cf. Tielens 2008). Disentangling these two scenarios is not easy observationally. Here we now consider the case where the PAH emission is assumed to come from within the shocked region. This scenario requires an efficient (re)formation route for PAHs in the diffuse ISM (see above). However, this is difficult to understand since PAHs are a product of high temperature chemistry involving abundant carbon bearing precursors such as $\mathrm{CH}_{4}$ and $\mathrm{C}_{2} \mathrm{H}_{2}$. In the low temperature diffuse ISM, an O-rich environment, these precursor species are never really very abundant. This conundrum is very reminiscent of the general problem of rapid dust destruction in the ISM and the long injection time scale for freshly formed dust (Dwek \& Scalo 1980; Draine \& Salpeter 1979; Jones et al. 1994, 1996). We note that, while dust grains may be rapidly covered by (thin)protective coatings between successive shock passages (cf. Tielens 1998), this is not a way out of this conundrum for PAHs. Perhaps, PAHs can be formed through prolonged photolysis of ice mantles accreted inside dense molecular clouds, although, presently, there is no experimental support for this suggestion. Lastly, observations have revealed the presence of PAHs associated with hot shocked gas in stellar (e.g., M 17) and galactic (e.g., M 82) wind regions (cf. Sect. 1). As will be discussed more extensively in MJT, these PAHs likely trace entrained cold gas which has not been fully exposed to the destructive effects of high velocity shocks.

For $v_{\mathrm{S}}<75 \mathrm{~km} \mathrm{~s}^{-1}$, PAH are disrupted only by inertial "sputtering" due to nuclear interactions. Serra Díaz-Cano \& Jones (2008) have studied the erosion of hydrogenated amorphous carbon $(\mathrm{a}-\mathrm{C}: \mathrm{H})$ arising from ion irradiation in shocks in the warm intercloud medium, using exactly the same shock profiles that we use for our PAH study, and it is interesting to note that, for a 50 carbon atom $\mathrm{PAH}$ molecule, the percentage of destruction is the same as for a-C:H, indicating that the lower density, more easily sputtered a-C:H and PAHs exhibit similar erosion characteristics in lower velocity shocks $\left(\simeq 125 \mathrm{~km} \mathrm{~s}^{-1}\right)$, as discussed in the previous section.

We now consider what happens to a PAH as a result of the ejection of aromatic carbon atoms, by the impacting ions, as a function of the fraction of carbon atoms removed from the structure. Our results show that ionic collisions severely modify PAH in shocks with velocities between $75-150 \mathrm{~km} \mathrm{~s}^{-1}$. Nuclear and electronic interaction lead to distinctly different molecular destruction routes. Specifically, electronic excitation (either by impacting ions or electrons) lead to a high vibrational excitation of the PAH and this PAH will relax by "losing" the weakest link in its skeleton. Initially, this will be the peripheral $\mathrm{H}$-atoms or other functional groups. For large PAHs (50-100 C-atoms), the resulting "pure-C" may quickly isomerize to very stable carbon cluster such as fullerenes. Fullerenes are very stable against $\mathrm{C}_{2}$ loss. Measured dissociation energies of fullerenes are in the range of 7 to $9.5 \mathrm{eV}$, with $\mathrm{C}_{60}$ itself at $E_{0}=9.8 \pm 0.1 \mathrm{eV}$ (Tomita et al. 2001).

In contrast, nuclear interaction will act on the $\mathrm{C}$-atom hit by the projectile ion. This will randomly remove $\mathrm{C}$-atoms from the $\mathrm{C}$-skeleton of the PAH. Some isomerization may occur if internal excitation energy is left behind. However, unless the C-atom loss in the shock is very large, likely this is insufficient to affect the overall PAH structure. In a simple "geometrical" analysis, if one randomly removes $\mathrm{C}$ atoms from a PAH with $N_{\mathrm{C}}=50$, in the absence of any annealing of the PAH chemical structure, we find that the loss of $10 \%$ of the $\mathrm{C}$ atoms leads to a loss of of the order of $\approx 50 \%$ of the aromatic character. However, the loss of $\approx 20 \%$ of the $\mathrm{C}$ atoms leads to the almost complete loss of aromatic character and to the onset of the fragmentation of the molecule. Figure 11 illustrates these effects and shows the PAH evolution following the loss of carbon atoms, $N_{\mathrm{C}}$ (lost), for the two limiting cases: 1) where there is an instantaneous and random removal of the lost carbon atoms (appropriate for nuclear interaction) and 2) where the carbon atoms are removed only from the periphery in order to preserve aromatic domain as much as possible (likely appropriate for electronic excitation through either impact ions or electrons). The reality of PAH erosion in shocks may well lie somewhere between these two extremes and may also involve isomerisation and the formation of five-fold carbon rings that distort the structure from a perfectly two-dimensional form. This then begs the question as to the exact form and structure of small carbon species once growth resumes by atom insertion and addition.

After the shock wave has passed, the resulting PAH can react chemically with impacting $\mathrm{H}, \mathrm{C}, \mathrm{O}$, and $\mathrm{N}$ atoms. 


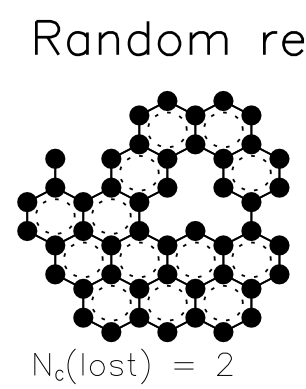

$\mathrm{V}_{\text {shock }}=50$

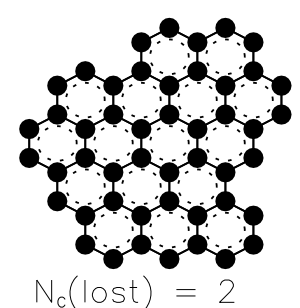

Minimal aromatic domain loss

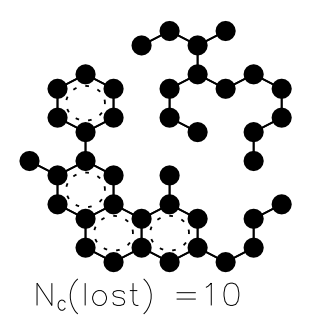

75

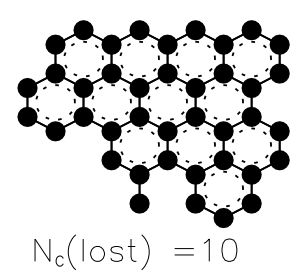

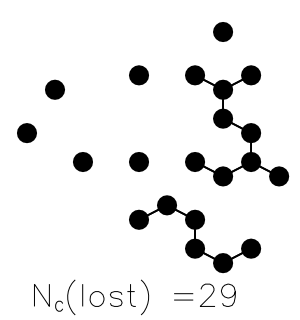

$125 \mathrm{~km} / \mathrm{s}$

100
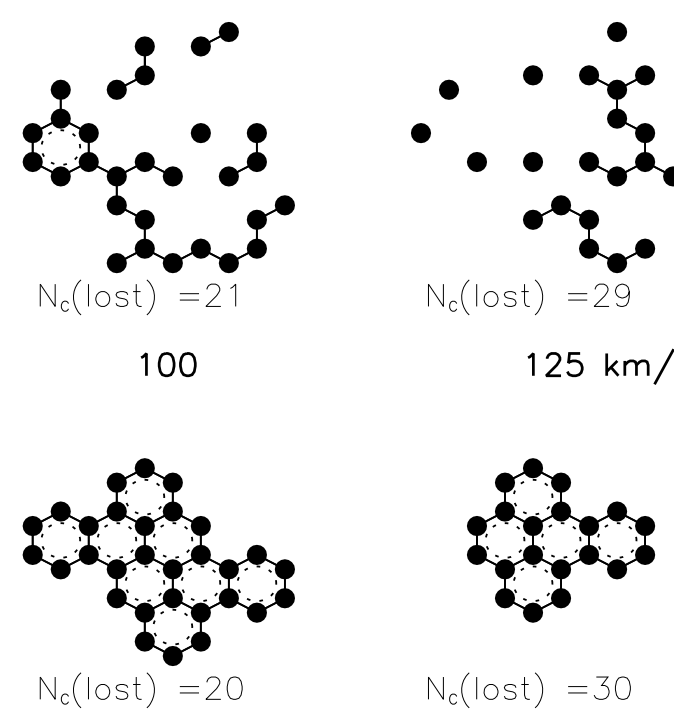
S

.

Fig. 11. The evolution of a 50 carbon atom PAH following the loss of $N_{\mathrm{C}}($ lost) carbon atoms, as a function of the shock velocity, for the two limiting cases: instantaneous and random removal of the lost carbon atoms (top row), and carbon atom removal only from the periphery of the molecule (bottom row).

\begin{abstract}
A distinction can be made between $\mathrm{H}$ and $\mathrm{O}$ in the one hand and $\mathrm{C}$ and $\mathrm{N}$ on the other hand, The latter can restore the stable aromatic character of the PAH skeleton while the former lead only to very unstable structures. Likely, then, $\mathrm{H}$ and $\mathrm{O}$ atom addition can be reversed by UV photon absorption without loss of $\mathrm{C}$ (Allamandola et al. 1989). Hence, the PAH carbon skeleton may be able to "repair" itself to an aromatic structure, possibly incorporating $\mathrm{N}$-atoms. The possible presence of $\mathrm{N}$-atoms deeply in the C-skeleton of PAHs in the diffuse medium (but not in C-dust birth sites such as C-rich planetary nebulae) has been inferred from the peak position of the $6.2 \mu \mathrm{m}$ band (Peeters et al. 2002). It has also been suggested that the $6.2 \mu \mathrm{m}$ band position variations can be explained by a varying aliphatic to aromatic carbon content in carbonaceous particles (Pino et al. 2008). The difference in molecular structure between circumstellar PAHs and the general ISM implies an active chemistry in the ISM that is able to insert $\mathrm{N}$ atoms deeply in the carbon skeleton. This is very puzzling given the very stable character of the aromatic backbone and the low temperature of the ISM. Here, we surmise that, unlike UV photolysis or chemical attack, nuclear interactions in interstellar shocks may be a viable pathway to promote $\mathrm{N}$-incorporations deep inside PAHs. However, given that the nitrogen abundance is a factor of a few lower than that for carbon - depending on the fraction of carbon remaining in dust and PAHs - carbon atom insertion ought to be favoured over that for nitrogen, in the absence of any chemically-selective route for nitrogen insertion.
\end{abstract}

\section{Conclusions}

We have extensively studied the effects of PAH processing by shocks with velocities between 50 and $200 \mathrm{~km} \mathrm{~s}^{-1}$, in terms of collisions with ions and electrons which can lead to carbon atom loss, with a consequent disruption and destruction of the molecule.
An ionic collision consists of two simultaneous processes which can be treated separately: a binary collisions between the projectile and one single atom in the target (nuclear interaction) and the energy loss to the atomic electrons (electronic interaction). For the nuclear interaction, we modified the existing theory in order to treat collisions able to transfer energy above a specific threshold. This is the case we are interested in, which has not been treated in previous studies. For electronic interaction and collisions with electrons we developed specific models for PAH, described in MJT.

The PAH dynamics in the shocks is evaluated using the same approach as in our previous work (Jones et al. 1994, 1996). For nuclear interaction, the level of carbon atom loss increases for decreasing values of the threshold energy $T_{0}$. We adopt $T_{0}=$ $7.5 \mathrm{eV}$ as a reasonable value, but experimental determinations are necessary. In ionic collisions, the carbon contribution to PAH destruction is totally negligible because of its very low abundance with respect to $\mathrm{H}$ and $\mathrm{He}$. The fractional destruction induced by nuclear excitation increases with the PAH size, while in case of electronic excitation and electron collisions is lower for higher $N_{\mathrm{C}}$ values, i.e. bigger PAHs are more resistant than the smaller ones against electron and electronic processing.

The parameter $E_{0}$ required for the evaluation of PAH destruction due to electron and electronic interaction is unfortunately not well constrained. We adopt a value of $4.58 \mathrm{eV}$ consistent with extrapolations to interstellar conditions, but better determinations would be desirable.

Electronic interaction, both inertial and thermal, plays a marginal role in PAH processing by shocks. We find that 50 carbon atoms PAHs are significantly disrupted in ionic collisions for shock velocities below $75 \mathrm{~km} \mathrm{~s}^{-1}$, mainly by inertial "sputtering" by helium during nuclear interaction. Our results indicate $5-15 \% \mathrm{C}$ atom loss, sufficient to cause a severe de-naturation of the PAH aromatic structure. Above $100 \mathrm{~km} \mathrm{~s}^{-1}$ such PAHs are instead totally destroyed by collisions with thermal electrons. 
For $N_{\mathrm{C}}=200$, PAHs experience increasing damaging caused by nuclear ionic collisions up to $100 \mathrm{~km} \mathrm{~s}^{-1}$, which turns into complete atomic loss for higher velocities. In this case the destruction is due to the combined effect of electrons and nuclear interaction with thermal ions.

The calculated $\mathrm{PAH}$ lifetime against destruction, $t_{\mathrm{SNR}}$, is $1.6 \times 10^{8} \mathrm{yr}$ and $1.4 \times 10^{8} \mathrm{yr}$ for $N_{\mathrm{C}}=50$ and 200 respectively. Small PAHs are preferentially destroyed by electrons, big PAHs by ions. The calculated lifetimes are smaller than the values found for carbonaceous grains $\left(6 \times 10^{8} \mathrm{yr}\right)$ but close to that for hydrogenated amorphous carbon $\left(2 \times 10^{8} \mathrm{yr}\right)$, and far from the stardust injection timescale of $2.5 \times 10^{9} \mathrm{yr}$. The presence of PAHs in shocked regions therefore requires an efficient reformation mechanism and/or a protective environment.

We surmise that the molecular structure of PAHs is strongly affected by shock processing in the interstellar medium. Electronic excitation by impacting ions or electrons may lead to isomerization into stable pure-C species such as fullerenes. In contrast, nuclear interaction may lead to the formation of $\mathrm{N}$-containing PAHs. Further laboratory studies are required to demonstrate the viability of these chemical routes.

\section{Appendix A: $S_{\mathrm{n}}, \sigma$ and $\langle T\rangle$}

A fundamental quantity to describe the nuclear scattering is the energy transfer cross section $\sigma(E, T)$, which is function of the kinetic energy $E$ of the projectile and of the energy $T$ transferred to the target by the projectile in a single collision. At low energies $(\varepsilon \lesssim 1)$, an approximated expression for the cross section can be calculated using the power approximation to the ThomasFermi model of interatomic interaction, i.e. with a potential of the form $V(r) \propto r^{-1 / m}$, where $r$ is the distance between colliding nuclei and $m$ is a parameter related to the steepness of the interatomic potential. The quantity $m$ can also be interpreted as an indicator of the energy of the projectile, varying slowly from $m=1$ at high energies to $m \approx 0$ at low energies (Lindhard et al. 1968; Winterbon et al. 1970). We have that

$\mathrm{d} \sigma(E, T) \cong C_{m} E^{-m} T^{-1-m} \mathrm{~d} T \quad 0 \lesssim T \lesssim T_{\mathrm{m}}$

with

$C_{m}=\frac{\pi}{2} \lambda_{m} a^{2}\left(\frac{M_{1}}{M_{2}}\right)^{m}\left(\frac{2 Z_{1} Z_{2} e^{2}}{a}\right)^{2 m}$

where $T_{\mathrm{m}}$ is the maximum transferable energy, corresponding to a head-on collision (impact parameter $p=0$ ). The dimensionless quantity $\lambda_{\mathrm{m}}=\lambda_{\mathrm{m}}(m)$ varies slowly from 0.5 for $m=1$ (high energy, i.e. pure Rutherford scattering, Simmons 1965), to 24 for $m=0$ (very low energy).

The power approximation of the Thomas-Fermi cross section (Eq. (A.1)) gives the following expression for the nuclear stopping cross section, obtained evaluating the integral in Eq. (4) between 0 and $T_{\mathrm{m}}$ :

$S_{\mathrm{n}}(E)=\frac{1}{1-m} C_{m} \gamma^{1-m} E^{1-2 m}$.

For Thomas-Fermi interaction, using Eqs. (1), (A.2), (3) and (A.3), this leads to

$S_{\mathrm{n}}(E)=4 \pi a Z_{1} Z_{2} e^{2} \frac{M_{1}}{M_{1}+M_{2}} s_{\mathrm{n}}(\varepsilon)$

with

$s_{\mathrm{n}}(\varepsilon)=\frac{\lambda_{m}}{2(1-m)} \varepsilon^{1-2 m}$.
For heavy screening $(\varepsilon \ll 1, m \cong 0)$ the accuracy of the Thomas-Fermi reduced stopping cross section $s_{\mathrm{n}}(\varepsilon)$ to reproduce the experimental data is at best a factor of two. For this reason we adopt instead the Universal reduced stopping cross section $s_{\mathrm{n}}^{\mathrm{U}}($ Eq. (5)) from Ziegler et al. (1985) with the appropriate screening length $a_{\mathrm{U}}$ (Eq. (2)), which provides a good fit to the experimental data at low energies as well.

The nuclear stopping cross section above threshold is given by Eq. (10). Remembering that $T_{\mathrm{m}}=\gamma E$ and $T_{0}=\gamma E_{0 \mathrm{n}}$, this can be rewritten as follows

$$
\begin{aligned}
S_{\mathrm{n}}(E) & =\frac{C_{m} E^{-m}}{1-m}\left[T_{\mathrm{m}}^{1-m}-T_{0}^{1-m}\right] \\
& =\frac{C_{m} E^{-m}}{1-m} T_{\mathrm{m}}^{1-m}-\frac{C_{m} E^{-m}}{1-m} T_{0}^{1-m} \\
& =\frac{C_{m} \gamma^{1-m}}{1-m} E^{1-2 m}-\frac{C_{m} \gamma^{1-m}}{1-m} E^{-m} E_{0 \mathrm{n}}^{1-m}
\end{aligned}
$$

The first term in the right side of the equation is equal to the nuclear stopping cross section in the no-threshold case $\left(T_{0}=0\right)$

$$
\frac{C_{m} \gamma^{1-m}}{1-m} E^{1-2 m}=4 \pi a Z_{1} Z_{2} e^{2} \frac{M_{1}}{M_{1}+M_{2}} s_{\mathrm{n}}(\varepsilon) \equiv S_{\mathrm{n}}^{0}(E) \text {. }
$$

In the second term we use the equality $E^{-m}=\left(E^{1-2 m} / E^{1-m}\right)$ to obtain

$$
\begin{aligned}
\frac{C_{m} \gamma^{1-m}}{1-m} E^{-m} E_{0 \mathrm{n}}^{1-m} & =\frac{C_{m} \gamma^{1-m}}{1-m} E^{1-2 m}\left(\frac{E_{0 \mathrm{n}}}{E}\right)^{1-m} \\
& =4 \pi a Z_{1} Z_{2} e^{2} \frac{M_{1}}{M_{1}+M_{2}} s_{\mathrm{n}}(\varepsilon) \times\left(\frac{E_{0 \mathrm{n}}}{E}\right)^{1-m} .
\end{aligned}
$$

Combining the two we obtain the following expression for $S_{\mathrm{n}}$

$S_{\mathrm{n}}(E)=4 \pi a Z_{1} Z_{2} e^{2} \frac{M_{1}}{M_{1}+M_{2}} S_{\mathrm{n}}(\varepsilon)\left[1-\left(\frac{E_{0 \mathrm{n}}}{E}\right)^{1-m}\right]$.

The total cross section is given by Eq. (11)

$$
\begin{aligned}
\sigma(E) & =\frac{C_{m} E^{-m}}{m}\left[T_{0}^{-m}-T_{\mathrm{m}}^{-m}\right] \\
& =C_{m} E^{-m} \frac{T_{0}^{-m}}{m}-C_{m} E^{-m} \frac{T_{\mathrm{m}}^{-m}}{m} .
\end{aligned}
$$

The term $\left(T_{0}^{-m} / m\right)$ can be rewritten as follows

$$
\begin{aligned}
\frac{T_{0}^{-m}}{m} & =\frac{T_{\mathrm{m}}^{1-m}}{1-m} \times \frac{1-m}{m} \times \frac{T_{0}^{-m}}{T_{\mathrm{m}}^{1-m}} \\
& =\frac{\gamma^{1-m} E^{1-m}}{1-m} \times \frac{1-m}{m} \times \frac{\gamma^{-m} E_{0 \mathrm{n}}^{-m}}{\gamma^{1-m} E^{1-m}} \\
& =\frac{\gamma^{1-m} E^{1-m}}{1-m} \times \frac{1-m}{m} \times \frac{1}{\gamma E}\left(\frac{E_{0 \mathrm{n}}}{E}\right)^{-m} .
\end{aligned}
$$

Then we have

$$
\begin{aligned}
C_{m} E^{-m} \frac{T_{0}^{-m}}{m} & =\frac{C_{m} \gamma^{1-m}}{1-m} E^{1-2 m} \times \frac{1-m}{m} \times \frac{1}{\gamma E}\left(\frac{E_{0 \mathrm{n}}}{E}\right)^{-m} \\
& =S_{\mathrm{n}}^{0}(E) \times \frac{1-m}{m} \times \frac{1}{\gamma E}\left(\frac{E_{0 \mathrm{n}}}{E}\right)^{-m} .
\end{aligned}
$$


Using a similar approach we can write the term $\left(T_{\mathrm{m}}^{-m} / m\right)$ as

$$
\begin{aligned}
\frac{T_{\mathrm{m}}^{-m}}{m} & =\frac{T_{\mathrm{m}}^{-m}}{m} \times \frac{1-m}{1-m} \times \frac{T_{\mathrm{m}}}{T_{\mathrm{m}}} \\
& =\frac{T_{\mathrm{m}}^{1-m}}{1-m} \times \frac{1-m}{m} \times \frac{1}{T_{\mathrm{m}}} \\
& =\frac{\gamma^{1-m} E^{1-m}}{1-m} \times \frac{1-m}{m} \times \frac{1}{\gamma E} .
\end{aligned}
$$

Then second term in the right side of the equation then becomes

$$
\begin{aligned}
C_{m} E^{-m} \frac{T_{\mathrm{m}}^{-m}}{m} & =\frac{C_{m} \gamma^{1-m}}{1-m} E^{1-2 m} \times \frac{1-m}{m} \times \frac{1}{\gamma E} \\
& =S_{\mathrm{n}}^{0}(E) \times \frac{1-m}{m} \times \frac{1}{\gamma E} .
\end{aligned}
$$

Combining the two terms we obtain for $\sigma(E)$ the final expression $\sigma(E)=4 \pi a Z_{1} Z_{2} e^{2} \frac{M_{1}}{M_{1}+M_{2}} s_{\mathrm{n}}(\varepsilon) \frac{1-m}{m} \frac{1}{\gamma E}\left[\left(\frac{E_{0 \mathrm{n}}}{E}\right)^{-m}-1\right]$.

The expression for the average transferred energy $\langle T(E)\rangle$ derives directly from Eq. (12) when applying the relations $T_{\mathrm{m}}=\gamma E$ and $T_{0}=\gamma E_{0 \mathrm{n}}$

$$
\begin{aligned}
\langle T(E)\rangle & =\frac{m}{1-m} \frac{T_{\mathrm{m}}^{1-m}-T_{0}^{1-m}}{T_{0}^{-m}-T_{\mathrm{m}}^{-m}} \\
& =\frac{m}{1-m} \frac{\gamma^{1-m}\left(E^{1-m}-E_{0 \mathrm{n}}^{1-m}\right)}{\gamma^{-m}\left(E_{0 \mathrm{n}}^{-m}-E^{-m}\right)} \\
& =\frac{m}{1-m} \gamma \frac{E^{1-m}-E_{0 \mathrm{n}}^{1-m}}{E_{0 \mathrm{n}}^{-m}-E^{-m}} .
\end{aligned}
$$

\section{Appendix B: Low and high energy regime above threshold}

In the low energy regime $m$ can be taken equal to 0 , so the differential cross section $\mathrm{d} \sigma$ becomes

$\mathrm{d} \sigma(E, T)=\cong \frac{\pi}{2} \lambda_{0} a^{2} T^{-1} \mathrm{~d} T$.

The corresponding expressions for $S_{\mathrm{n}}(E), \sigma(E)$ and $\langle T(E)\rangle$ above threshold are calculated from Eqs. (7)-(9) respectively

$$
\begin{aligned}
& S_{\mathrm{n}}(E)=\frac{\pi}{2} \lambda_{0} a^{2} \gamma\left(E-E_{0 \mathrm{n}}\right) \\
& \sigma(E)=\frac{\pi}{2} \lambda_{0} a^{2} \ln \frac{E}{E_{0 \mathrm{n}}} \\
& \langle T(E)\rangle=\gamma\left(E-E_{0 \mathrm{n}}\right)\left(\ln \frac{E}{E_{0 \mathrm{n}}}\right)^{-1} .
\end{aligned}
$$

In the high enery regime $m=1$, then we have

$\mathrm{d} \sigma(E, T) \cong \frac{M_{1}}{M_{2}} Z_{1}^{2} Z_{2}^{2} e^{4} \pi \frac{1}{E} T^{-2} \mathrm{~d} T$ and consequently

$$
\begin{aligned}
& S_{\mathrm{n}}(E)=\frac{M_{1}}{M_{2}} Z_{1}^{2} Z_{2}^{2} e^{4} \pi \frac{1}{E} \ln \frac{E}{E_{0 \mathrm{n}}} \\
& \sigma(E)=\frac{M_{1}}{M_{2}} Z_{1}^{2} Z_{2}^{2} e^{4} \pi \frac{1}{E} \frac{1}{\gamma E_{0 \mathrm{n}}} \\
& \langle T(E)\rangle=\gamma E_{0 \mathrm{n}} \ln \frac{E}{E_{0 \mathrm{n}}} .
\end{aligned}
$$

\section{Appendix C: Orientation correction}

To calculate the orientation correction factor $\langle S\rangle$, let us consider the two versors $\boldsymbol{u}$ and $\boldsymbol{n}$, perpendicular to the PAH surface and forming between each other the angle $\vartheta$. This configuration defines two possible orientations for the molecule. The average orientation is then given by the following integral:

$\langle S\rangle=\frac{1}{2 \pi} \int \boldsymbol{u} \cdot \boldsymbol{n} \mathrm{d} \Omega$

where $\mathrm{d} \Omega=\sin \vartheta \mathrm{d} \vartheta \mathrm{d} \varphi$ is the solid angle element and $\boldsymbol{u} \cdot \boldsymbol{n}=\cos \vartheta$ is the scalar product between the two versors. With these substitutions we obtain

$\langle S\rangle=\frac{1}{2 \pi} \int_{\varphi=0}^{2 \pi} \mathrm{d} \varphi \int_{\vartheta=0}^{\pi / 2} \cos \vartheta \sin \vartheta \mathrm{d} \vartheta=\frac{1}{2}$

Acknowledgements. We are grateful to L. Allamandola and L. Verstraete for useful discussions, and we acknowledge our referee Tom Hartquist for careful reading and helpful comments. E.R.M. thanks G. Lavaux for support and technical assistance and acknowledges financial support by the EARA Training Network (EU grant MEST-CT-2004-504604).

\section{References}

Allamandola, L. J., Tielens, A. G. G. M., \& Barker, J. R. 1989, ApJS, 71, 733 Andersen, M., Rho, J., Hewitt, J., \& Reach, W. 2007, in BAAS, 38, 932 Armus, L., Draine, B., Engelbracht, C., et al. 2007, in BAAS, 38, 789 Banhart, F. 1997, J. Appl. Phys., 81, 3440

Berné, O., Joblin, C., Deville, Y., et al. 2007, A\&A, 469, 575 Borkowski, K. J., \& Dwek, E. 1995, ApJ, 454, 254

Bregman, J. D., Allamandola, L. J., Witteborn, F. C., Tielens, A. G. G. M., \& Geballe, T. R. 1989, ApJ, 344, 791

Bregman, J. N. 1980, ApJ, 236, 577

Cardelli, J. A., Meyer, D. M., Jura, M., \& Savage, B. D. 1996, ApJ, 467, 334

Cherchneff, I., Barker, J. R., \& Tielens, A. G. G. M. 1992, ApJ, 401, 269

Cosslett, V. E. 1978, J. Microsc., 113, 113

Désert, F.-X., Boulanger, F., \& Puget, J. L. 1990, A\&A, 237, 215

Draine, B. T., \& Li, A. 2001, ApJ, 551, 807

Draine, B. T., \& Salpeter, E. E. 1979, ApJ, 231, 438

Dwek, E., \& Scalo, J. M. 1980, ApJ, 239, 193

Frenklach, M., \& Feigelson, E. D. 1989, ApJ, 341, 372

Füller, T., \& Banhart, F. 1996, Chem. Phys. Lett., 254, 372

Guillet, V., Pineau Des Forêts, G., \& Jones, A. P. 2007, A\&A, 476, 263

Irwin, J. A., \& Madden, S. C. 2006, A\&A, 445, 123

Irwin, J. A., Kennedy, H., Parkin, T., \& Madden, S. 2007, A\&A, 474, 461 Jochims, H. W., Ruhl, E., Baumgartel, H., Tobita, S., \& Leach, S. 1994, ApJ, 420, 307

Jones, A. P., Tielens, A. G. G. M., Hollenbach, D. J., \& McKee, C. F. 1994, ApJ, 433, 797

Jones, A. P., Tielens, A. G. G. M., \& Hollenbach, D. J. 1996, ApJ, 469, 740 Latter, W. B. 1991, ApJ, 377, 187

Le Page, V., Snow, T. P., \& Bierbaum, V. M. 2001, ApJS, 132, 233

Lindhard, J., Scharff, M., \& Schiott, H. E. 1963, Mat. Fys. Medd. Dan. Vid. Selsk., 33, 14

Lindhard, J., Nielsen, V., \& Scharff, M. 1968, Mat. Fys. Medd. Dan. Vid. Selsk., 36,10 
E. R. Micelotta et al.: PAH processing in interstellar shocks

McKee, C. 1989, in Interstellar Dust, ed. L. J. Allamandola, \& A. G. G. M. Tielens, IAU Symp., 135, 431

McKee, C. F., \& Ostriker, J. P. 1977, ApJ, 218, 148

McKee, C. F., Hollenbach, D. J., Seab, G. C., \& Tielens, A. G. G. M. 1987, ApJ, 318,674

Micelotta, E. R., Jones, A. P., \& Tielens, A. G. G. M. 2010, A\&A, 510, A37 (MJT)

Montet, G. L. 1967, Carbon, 5, 19

Montet, G. L., \& Myers, G. E. 1971, Carbon, 9, 179

Nakai, K., Kinoshita, C., \& Matsunaga, A. 1991, Ultramicroscopy, 39, 361

O’Connor, D. J., \& Biersack, J. P. 1986, Nucl. Instrum. Methods Phys. Res. B, 15,14

O'Halloran, B., Satyapal, S., \& Dudik, R. P. 2006, ApJ, 641, 795

O'Halloran, B., Madden, S. C., \& Abel, N. P. 2008, ApJ, 681, 1205

Omont, A. 1986, A\&A, 164, 159

Peeters, E., Hony, S., Van Kerckhoven, C., et al. 2002, A\&A, 390, 1089

Podio, L., Bacciotti, F., Nisini, B., et al. 2006, A\&A, 456, 189

Pino, T., Dartois, E., Cao, A.-T., et al. 2008, A\&A, 490, 665

Rapacioli, M., Joblin, C., \& Boissel, P. 2005, A\&A, 429, 193

Serra Díaz-Cano, L., \& Jones, A. P. 2008, A\&A, 492, 127
Sigmund, P. 1981, Sputtering by Particle Bombardment, 1, ed. R. Behrisch, in Topics in Applied Physics (Springer-Verlag), 47

Simmons, J. H. W. 1965, Radiation Damage in Graphite (Pergamon Press)

Slavin, J. D. 2008, Space Sci. Rev., 50

Smith, J. D. T., Rudnick, L., Delaney, T., et al. 2009, ApJ, 693, 713

Sofia, U. J. 2009, in Cosmic Dust - Near and Far, ed. T. Henning, E. Grun, \& J. Steinacker

Tappe, A., Rho, J., \& Reach, W. T. 2006, ApJ, 653, 267

Tielens, A. G. G. M. 1998, ApJ, 499, 267

Tielens, A. G. G. M. 2005, The Physics and Chemistry of the Interstellar Medium (University of Cambridge Press)

Tielens, A. G. G. M. 2008, ARA\&A, 46, 289

Tomita, S., Andersen, J. U., Gottrup, C., Hvelplund, P., \& Pedersen, U. V. 2001, Phys. Rev. Lett., 87, 073401

Welty, D. E., Jenkins, E. B., Raymond, J. C., Mallouris, C., \& York, D. G. 2002, ApJ, 579, 304

Winterbon, K. B., Sigmund, P., \& Sanders, J. B. 1970, Mat. Fys. Medd. Dan. Vid. Selsk., 37, 14

Ziegler, J. F., Biersack, J. P., \& Littmark, U. 1985, The Stopping and Ranges of Ions in Matter, ed. J. F. Ziegler (Pergamon Press), 1 